\title{
Parabolic equations and the bounded slope condition
}

\author{
Verena Bögelein ${ }^{\mathrm{a}}$, Frank Duzaar ${ }^{\mathrm{b}}$, Paolo Marcellini $^{\mathrm{c}}$, Stefano Signoriello ${ }^{\mathrm{b}}$ \\ ${ }^{a}$ Fachbereich Mathematik, Universität Salzburg, Hellbrunner Str. 34, 5020 Salzburg, Austria \\ b Department Mathematik, Universität Erlangen-Nürnberg, Cauerstrasse 11, 91058 Erlangen, Germany \\ c Dipartimento di Matematica e Informatica "U.Dini”, Università di Firenze, Viale Morgagni 67/A, 50134 Firenze, Italy
}

Received 1 March 2015; received in revised form 22 November 2015; accepted 8 December 2015

Available online 23 December 2015

\begin{abstract}
In this paper we establish the existence of Lipschitz-continuous solutions to the Cauchy Dirichlet problem of evolutionary partial differential equations

$$
\left\{\begin{array}{c}
\partial_{t} u-\operatorname{div} D f(D u)=0 \quad \text { in } \Omega_{T}, \\
u=u_{o} \quad \text { on } \partial_{\mathcal{P}} \Omega_{T} .
\end{array}\right.
$$

The only assumptions needed are the convexity of the generating function $f: \mathbb{R}^{n} \rightarrow \mathbb{R}$, and the classical bounded slope condition on the initial and the lateral boundary datum $u_{o} \in W^{1, \infty}(\Omega)$. We emphasize that no growth conditions are assumed on $f$ and that - an example which does not enter in the elliptic case $-u_{o}$ could be any Lipschitz initial and boundary datum, vanishing at the boundary $\partial \Omega$, and the boundary may contain flat parts, for instance $\Omega$ could be a rectangle in $\mathbb{R}^{n}$.
\end{abstract}

() 2015 Published by Elsevier Masson SAS.

MSC: 35A01; 35K61; 35K86; 49J40

Keywords: Existence; Parabolic equations; Bounded slope condition; Lipschitz solutions

\section{Introduction}

Throughout the paper let $\Omega \subset \mathbb{R}^{n}$ be a bounded, open and convex set and $f: \mathbb{R}^{n} \rightarrow \mathbb{R}$ be a convex integrand. We define the energy functional $F: W^{1, \infty}(\Omega) \rightarrow \mathbb{R}$ by means of

$$
F(v):=\int_{\Omega} f(D v) \mathrm{d} x .
$$

A by now classical result, following the pioneering paper by Haar [15] and then Hartman-Nirenberg [16], Stampacchia [32], Miranda [27], Hartman-Stampacchia [17], which can nowadays be retrieved from textbooks (cf. [14, Chapter 1]),

E-mail addresses: verena.boegelein@sbg.ac.at (V. Bögelein), duzaar@math.fau.de (F. Duzaar), marcellini@math.unifi.it (P. Marcellini), Stefano.Signoriello@math.uni-erlangen.de (S. Signoriello). 
ensures to given boundary values $U_{o}: \partial \Omega \rightarrow \mathbb{R}$ satisfying the bounded slope condition the existence of a Lipschitz continuous minimizer $u$ to the variational functional $F$ with boundary values $U_{o}$. We refer to [8-11,22-25] for more recent research in the context of existence of Lipschitz solutions - bounded slope condition - classical Calculus of Variations. Surprisingly enough, a time dependent counterpart to this semi-classical theory is to our knowledge not known so far. Instead, many sophisticated techniques (such as Galerkin methods, monotone operators, nonlinear semigroup theory, etc.) lead to a huge variety of different existence results. However, the construction of Lipschitz. continuous solutions to evolutionary equations related to general convex integrands $f$ (without any further assumption on the growth of the integrand) does not fall into the range of these theories, and remained an open problem.

The aim of the present paper is to close this gap in the existence theory for parabolic equations. We present the corresponding parabolic analogue of the above mentioned elliptic existence result, i.e. the existence of a unique classical solution $u \in L^{\infty}\left(\Omega_{T}\right) \cap C^{0}\left([0, T] ; L^{2}(\Omega)\right)$ with Lipschitz continuous spatial gradient $D u \in L^{\infty}\left(\Omega_{T}, \mathbb{R}^{n}\right)$ to the following Cauchy-Dirichlet problem

$$
\left\{\begin{array}{c}
\partial_{t} u-\operatorname{div} D f(D u)=0, \quad \text { in } \Omega_{T}, \\
u=u_{o} \quad \partial_{\mathcal{P}} \Omega_{T},
\end{array}\right.
$$

will be established for any given Lipschitz continuous initial datum $u_{o}$ on $\Omega$, whose restriction to the boundary $\partial \Omega$ satisfies the bounded slope condition. Here, and in the following, the parabolic boundary of the space-time cylinder $\Omega_{T}:=\Omega \times(0, T)$ is defined by

$$
\partial_{\mathcal{P}} \Omega_{T}:=(\bar{\Omega} \times\{0\}) \cup(\partial \Omega \times(0, T)) .
$$

Prominent examples of variational integrands which are included in this framework are the area integrand

$$
f(\xi)=\sqrt{1+|\xi|^{2}}
$$

integrands with exponential growth, such as

$$
f(\xi)=\exp |\xi|^{2}
$$

or Orlicz type functionals like

$$
f(\xi):=|\xi| \log (1+|\xi|) .
$$

Any other convex function $f(\xi)$ not necessarily depending on the modulus of $\xi$, without any growth assumption, enters in our theory.

\subsection{The class of parabolic Lipschitz solutions}

In the parabolic case the formulation of Lipschitz continuous variational solutions accesses certain classes of functions, which can be interpreted as the parabolic analogue of the Lipschitz functions from the stationary case. In this circumstance we use the identification between the space of Lipschitz continuous functions $C^{0,1}(\Omega)$ and the Sobolev space $W^{1, \infty}(\Omega)$; see Proposition 2.1. Firstly, we define the class

$$
K\left(\Omega_{T}\right):=\left\{v \in L^{\infty}\left(\Omega_{T}\right) \cap C^{0}\left([0, T] ; L^{2}(\Omega)\right): D v \in L^{\infty}\left(\Omega_{T}, \mathbb{R}^{n}\right)\right\} .
$$

Next, for given $L \in(0, \infty)$ the class $K^{(L)}\left(\Omega_{T}\right)$ is defined by

$$
K^{(L)}\left(\Omega_{T}\right):=\left\{v \in K\left(\Omega_{T}\right):\|D v\|_{L^{\infty}\left(\Omega_{T}, \mathbb{R}^{n}\right)} \leq L\right\} .
$$

In the sequel we write $K^{(L)}$ for $L$ in the whole range $(0, \infty]$, with the meaning that $K^{(\infty)}:=K$. Now, given $u_{o} \in W^{1, \infty}(\Omega)$ and $L$ such that $\left\|D u_{o}\right\|_{L^{\infty}\left(\Omega, \mathbb{R}^{n}\right)} \leq L$, the subclasses $K_{u_{o}}^{(L)}\left(\Omega_{T}\right)$ then consist of those $v \in K^{(L)}\left(\Omega_{T}\right)$ coinciding with $u_{o}$ on the lateral boundary $\partial \Omega \times(0, T)$. Observe that for a.e. $t \in(0, T)$ the restriction $v(t):=v(\cdot, t)$ of $v \in K\left(\Omega_{T}\right)$ to the time slice $\Omega \times\{t\}$ is of class $W^{1, \infty}(\Omega)$. This allows to define the trace in the classical sense on these good times slices. Following an idea by Lichnewsky \& Temam [20], that was first used in the context of the evolutionary parametric minimal surface equation, the natural formulation of (1.2) can be given in terms of variational solutions. 
Definition 1.1 (Variational Solutions). Assume that $u_{o} \in W^{1, \infty}(\Omega)$. A map $u \in K_{u_{o}}\left(\Omega_{T}\right)$, with $T \in(0, \infty)$, is called a variational solution on $\Omega_{T}$ to the Cauchy-Dirichlet problem (1.2) if and only if the variational inequality

$$
\begin{aligned}
\iint_{\Omega_{T}} f(D u) \mathrm{d} x \mathrm{~d} t \leq & \iint_{\Omega_{T}}\left[\partial_{t} v(v-u)+f(D v)\right] \mathrm{d} x \mathrm{~d} t \\
& +\frac{1}{2}\left\|v(0)-u_{o}\right\|_{L^{2}(\Omega)}^{2}-\frac{1}{2}\|(v-u)(T)\|_{L^{2}(\Omega)}^{2}
\end{aligned}
$$

holds true for any $v \in K_{u_{o}}\left(\Omega_{T}\right)$ with $\partial_{t} v \in L^{2}\left(\Omega_{T}\right)$. Finally, a map $u \in K_{u_{o}}\left(\Omega_{\infty}\right)$ is termed a global variational solution (or variational solution on $\Omega_{\infty}$ ) if $u$ is a variational solution on $\Omega_{T}$ for all $T>0$.

\subsection{The main results}

Our main result concerning the existence of Lipschitz (with respect to the spatial variable) solutions to the CauchyDirichlet problem is the following:

Theorem 1.2 (Existence of Lipschitz solutions). Let $\Omega \subset \mathbb{R}^{n}$ be a bounded, open and convex set and suppose that $f: \Omega \rightarrow \mathbb{R}$ is a convex function, that $u_{o} \in W^{1, \infty}(\Omega)$ and that $U_{o}:=\left.u_{o}\right|_{\partial \Omega}$ satisfies the bounded slope condition (see Definition 2.2 below) for some constant $Q$. Then, there exists a unique global variational solution $u$ on $\Omega_{\infty}$ in the sense of Definition 1.1, satisfying the following gradient bound:

$$
\|D u\|_{L^{\infty}\left(\Omega_{\infty}, \mathbb{R}^{n}\right)} \leq \max \left\{Q,\left\|D u_{o}\right\|_{L^{\infty}\left(\Omega, \mathbb{R}^{n}\right)}\right\} .
$$

Moreover, if $f$ is of class $C^{1}$, then $u$ is the weak solution to the Cauchy Dirichlet problem (1.2).

We emphasize that the variational solution is unique even if the integrand $f$ is convex, but not strictly convex. This includes the case of the total variation, i.e. the integrand $f(\xi)=|\xi|$ is included in the theorem (apart form the final assertion concerning the weak form of the parabolic equation).

The existence result is completed by the following assertions on regularity properties of variational solutions.

Theorem 1.3 (Regularity of variational solutions). Suppose that $f: \Omega \rightarrow \mathbb{R}$ is convex and that $u_{o} \in W^{1, \infty}(\Omega)$. Then, the variational solution in the sense of Definition 1.1 on $\Omega_{T}, T \in(0, \infty]$, satisfies

$$
\partial_{t} u \in L^{2}\left(\Omega_{T}\right) \quad \text { and } \quad u \in C^{0, \frac{1}{2}}\left([0, \tau] ; L^{2}(\Omega)\right) \quad \forall \tau \in \mathbb{R} \cap(0, T] .
$$

Further, for the time derivative $\partial_{t} u$ there holds the quantitative $L^{2}$-bound

$$
\iint_{\Omega_{T}}\left|\partial_{t} u\right|^{2} \mathrm{~d} x \mathrm{~d} t \leq 2|\Omega| \sup _{B_{L}(0)}|f|,
$$

where $L \geq\|D u\|_{L^{\infty}\left(\Omega_{T}, \mathbb{R}^{n}\right)}$. If $f$ is of class $C^{1}$, then $u \in C^{0 ; 1,1 / 2}\left(\Omega_{T}\right)$.

Finally, if the integrand $f$ is more regular, then also variational solutions are more regular. For a short discussion on this subject we refer to $\S 8$.

We note that in general it is not possible to treat functionals depending on $x$ and $u$ within this context, because $u(x)$ and $u(x+h)$ must be at the same time parabolic minimizers of the same functional; also $u(x)+$ const, for different values of the constant, must have this property. This rules out general assumptions on the integrand. However in the elliptic case the treatment of some special cases has been possible; cf. [17,22]. We could then expect that similar results hold in the parabolic setting too.

\subsection{The method of proof}

The proof of the classical elliptic result (see for example [14]) is divided into three steps. The first step consists in minimizing the functional $F$ in classes of Lipschitz functions coinciding with the given boundary values on $\partial \Omega$ 
and possessing a Lipschitz constant below a given fixed number $L$. This can be viewed as the solution of an obstacle problem, where the obstacle is given by a gradient constraint. In general the solutions $u^{(L)}$ of this gradient constrained obstacle problem are not minimizing among all Lipschitz functions (without the constraint). However, if $\operatorname{Lip} u^{(L)}<L$, then $u^{(L)}$ minimizes $F$. The proof of this fact is the content of the second step. In the third step, the bounded slope condition with a constant $Q$ is used to ensure that the unique minimizers $u^{(L)}$, i.e. the minimizers to the gradient constrained obstacle problems with threshold $L>Q$, satisfy the gradient bound $\operatorname{Lip} u^{(L)} \leq Q$ and therefore are the unique minimizers among all Lipschitz functions satisfying the prescribed Dirichlet boundary condition.

Maybe, the purely nature of this variational approach was the reason why a corresponding parabolic analogue was not derived. This is quite surprising, since a variational formulation of parabolic problems as variational solutions, an idea going back to the work of Lichnewsky \& Temam [20], was known in the literature since 1978. However, even with the notion of variational solutions at hands, the theories (time independent versus time dependent) do not seem to have common features. The missing link (connecting the theories) can be found in the formulation of a conjecture going back to De Giorgi [13] on the existence of global weak solutions to the Cauchy problem for nonlinear hyperbolic wave equations, and of Ilmanen's proof of Brakke's existence theorem for motion by mean curvature via elliptic regularization; see [18]. In the latter case solutions to Brakke's mean curvature flow are constructed as limits of translative solutions of a variational problem involving the time variable as a further independent variable. De Giorgi suggested to establish the existence of solutions to nonlinear wave equations by means of limits of minimizers of convex variational integrals on $\mathbb{R}^{n} \times(0, \infty)$. That this conjecture holds true for wave equations with super-critical nonlinearity of the type $u_{t t}-\Delta u=|u|^{q-2} u$ at least up to subsequences was proved by Serra and Tilli in [30]. A related approach has been used in $[4,5]$ to treat evolutionary problems with variational structure. Similar results form a more abstract point of view have been obtained in $[1,2,26]$; see also the references therein.

In this paper we present a purely variational approach that utilizes the method of elliptic regularization. More precisely we consider variational functionals

$$
F_{\varepsilon}(v):=\iint_{\Omega_{T}} \mathrm{e}^{-t / \varepsilon}\left[\frac{1}{2}\left|\partial_{t} v\right|^{2}+\frac{1}{\varepsilon} f(D v)\right] \mathrm{d} x \mathrm{~d} t
$$

on classes of functions $v: \Omega_{T} \rightarrow \mathbb{R}$ defined on the whole space-time cylinder $\Omega_{T}$ satisfying the boundary condition $v=u_{o}$ on the parabolic boundary $\partial_{\mathcal{P}} \Omega_{T}$, while on $\Omega \times\{T\}$ no boundary values are prescribed. Note, that $F_{\varepsilon}(v)$ is strictly convex on the class of functions satisfying the initial condition $v(0)=u_{o}$. As can easily be seen, there are close ties between our functional $F_{\varepsilon}$ and the functionals defined in the works of De Giorgi and Ilmanen. The heuristics behind the approximation of solutions via elliptic regularization can most easily be explained by formally computing the Euler-Lagrange equation associated to $F_{\varepsilon}$. The computation shows that minimizers $u_{\varepsilon}$ of $F_{\varepsilon}$ formally fulfill the elliptic equation

$$
-\varepsilon \partial_{t t} u_{\varepsilon}+\partial_{t} u_{\varepsilon}-\operatorname{div} D f\left(D u_{\varepsilon}\right)=0 \quad \text { in } \Omega_{T} .
$$

Therefore it is natural to expect, that minimizers $u_{\varepsilon}$ converge (possible after passing to a subsequence) to a solution of the Cauchy-Dirichlet problem (1.2). This indicates a possible strategy for a proof of Theorem 1.2. Since we are interested in Lipschitz solutions, the method has to be adjusted. In a first step, one would like to construct variational solutions satisfying a gradient constraint. Here we use the method of elliptic regularization. We minimize $F_{\varepsilon}$ in the class $K_{u_{o}}^{(L)}\left(\Omega_{T}\right)$, provided this class is non-empty. This is for example fulfilled if $L$ is chosen large enough, i.e. $L \geq\left\|D u_{o}\right\|_{L^{\infty}(\Omega)}$, in which case the time independent extension of $u_{o}$ to the whole cylinder $\Omega_{T}$ is admissible. Since solutions to obstacle problems in general will not solve the Euler-Lagrange equation, the heuristics from above has to be carried out on the level of minimizers. This can be achieved by proving certain energy bounds ensuring the sub-convergence of the sequence of minimizers $u_{\varepsilon}$ to some limit function $u^{(L)}$. Using the minimality of $u_{\varepsilon}$ by direct comparison arguments allows to establish that $u^{(L)}$ is a solution of the gradient constrained problem.

The second step consists - as in the elliptic case - in the observation that a variational solution $u^{(L)}$ whose $L^{\infty}$-norm of the spatial gradient is strictly less than the threshold $L$, actually solves the unconstrained problem in the sense of Definition 1.1. This follows by a direct comparison argument. With this respect, it is natural to seek for geometric conditions guaranteeing an a priori gradient bound. It is exactly this point where the classical bounded slope condition enters the theory. Utilizing a comparison principle for variational solutions it is possible to show that the constrained minimizers $u^{(L)}$ in $K_{u_{o}}^{(L)}\left(\Omega_{T}\right)$ with $L>Q$, where $Q$ is the constant from the bounded slope condition, 
satisfy the gradient bound $\left\|D u^{(L)}\right\|_{L^{\infty}\left(\Omega_{T}, \mathbb{R}^{n}\right)} \leq Q$. Together with the previous observation, this proves that $u^{(L)}$ is the desired variational solution. In case of a differentiable integrand $f$ one can pass to the Euler-Lagrange equation. Suitable Poincaré type inequalities (in the interior, the lateral boundary, the initial time, and the intersection of the latter two) which can be retrieved for instance from [6,7], imply that the variational solution belongs to a certain parabolic Morrey-space. A classical result of Da Prato then yields that $u \in C^{0 ; 1, \frac{1}{2}}\left(\bar{\Omega}_{T}\right)$, i.e. the solution is Lipschitzcontinuous with respect to the parabolic metric. Higher regularity can be shown by standard arguments (the parabolic De Giorgi-Nash-Moser theory and bootstrap arguments), provided $f$ is higher regular.

\section{Notations and preliminaries}

\subsection{Notations}

For $p \in[1, \infty], n \in \mathbb{N}$ and an open set $\Omega \subset \mathbb{R}^{n}$, the spaces $L^{p}(\Omega), W^{1, p}(\Omega)$ and $W_{0}^{1, p}(\Omega)$ denote the usual Lebesgue and Sobolev spaces, respectively. Moreover, for $T \in(0, \infty]$, by $\Omega_{T}$ we denote the space-time cylinder $\Omega \times(0, T)$. Further, for a set $A$ the characteristic function of $A$ shall be denoted by $\chi_{A}$. Throughout the paper we often use the identification of the class of Lipschitz continuous functions $C^{0,1}(\bar{\Omega})$ with the space $W^{1, \infty}(\Omega)$. For later usage we recall the definition of the Lipschitz constant of a continuous function $u$ on $\bar{\Omega}$ :

$$
[u]_{0,1 ; \Omega}:=\sup _{x \neq y, x, y \in \Omega} \frac{|u(x)-u(y)|}{|x-y|}<\infty .
$$

The connection between the spaces $C^{0,1}(\bar{\Omega})$ and $W^{1, \infty}(\Omega)$ is given in the following result:

Proposition 2.1. Let $\Omega \subset \mathbb{R}^{n}$ be a domain. A map $u \in L_{\mathrm{loc}}^{\infty}(\Omega)$ admits a weak derivative $D u \in L^{\infty}\left(\Omega, \mathbb{R}^{n}\right)$ if and only if $u$ is Lipschitz continuous on $\Omega$ with respect to the inner metric $\mathbf{d}_{\Omega}$. In this case the (optimal) Lipschitz constant $[u]_{0,1 ; \Omega}$ is given by $\|D u\|_{L^{\infty}\left(\Omega, \mathbb{R}^{n}\right)}$.

In the case of a convex domain $\Omega$ the inner metric is the Euclidean distance, i.e. in this case we have $\mathbf{d}_{\Omega}(x, y) \equiv$ $|x-y|$ for any $x, y \in \Omega$. For a nonconvex set $\mathbf{d}_{\Omega}$ is the minimal distance inside the set.

\subsection{Bounded slope condition}

As in the case of stationary minimization problems the bounded slope condition plays a fundamental role, since its validity guarantees suitable affine comparison functions. These affine functions can be used in the comparison principle, yielding pointwise estimates from above and below. The precise definition is as follows:

Definition 2.2. We say that a function $U: \partial \Omega \rightarrow \mathbb{R}$ satisfies the bounded slope condition with constant $Q>0$ if for any $x_{o} \in \partial \Omega$ there exist two affine functions $w_{x_{o}}^{-}$and $w_{x_{o}}^{+}$with $\left[w_{x_{o}}^{-}\right]_{0,1} \leq Q$ and $\left[w_{x_{o}}^{+}\right]_{0,1} \leq Q$ such that

$$
w_{x_{o}}^{-}(x) \leq U(x) \leq w_{x_{o}}^{+}(x), \quad \text { for any } x \in \partial \Omega
$$

and $w_{x_{o}}^{-}\left(x_{o}\right)=U\left(x_{o}\right)=w_{x_{o}}^{+}\left(x_{o}\right)$ holds true.

If $\Omega$ is a uniformly convex, bounded $C^{2}$-domain and $v \in C^{2}\left(\mathbb{R}^{n}\right)$, then $\left.v\right|_{\partial \Omega}$ satisfies the bounded slope condition; for more details see [14,27]. A completely different example, which has no correspondence in the elliptic case and which is covered in this context, is a Lipschitz initial boundary datum $u_{o}$ defined on a rectangle in $\mathbb{R}^{n}$ vanishing at the boundary; i.e. the boundary of our set can contain flat pieces.

The following Lemma shows that, given a Lipschitz continuous function whose restriction to the boundary satisfies the bounded slope condition, we can tilt the affine functions from the bounded slope condition in such a way that, on the one hand the bounded slope condition holds true with a larger constant, and on the other hand the function itself is squeezed on the whole domain between two affine functions. 
Lemma 2.3. Let $u_{o}: \Omega \rightarrow \mathbb{R}$ with $\left[u_{o}\right]_{0,1} \leq Q_{1}$ such that $U:=\left.u_{o}\right|_{\partial \Omega}$ satisfies the bounded slope condition with constant $Q_{o}$. Then for any boundary point $x_{o} \in \partial \Omega$ there exist two affine functions $w_{x_{o}}^{-}$and $w_{x_{o}}^{+}$with $\left[w_{x_{o}}^{-}\right]_{0,1} \leq$ $\max \left\{Q_{o}, Q_{1}\right\}$ and $\left[w_{x_{o}}^{+}\right]_{0,1} \leq \max \left\{Q_{o}, Q_{1}\right\}$ such that

$$
w_{x_{o}}^{-}(x) \leq u_{o}(x) \leq w_{x_{o}}^{+}(x), \quad \text { for any } x \in \bar{\Omega}
$$

and $w_{x_{o}}^{-}\left(x_{o}\right)=U\left(x_{o}\right)=w_{x_{o}}^{+}\left(x_{o}\right)$ holds true.

Proof. Consider $u: \Omega \rightarrow \mathbb{R}$ with $[u]_{0,1} \leq Q_{1}$ such that $U:=\left.u\right|_{\partial \Omega}$ satisfies the bounded slope condition with constant $Q_{o}$. Let $w_{x_{o}}^{+}$denote an affine function - which exists since $U$ satisfies the bounded slope condition - coinciding with $U$ at $x_{o}$ with $\left[w_{x_{o}}^{+}\right]_{0,1} \leq Q$ and $U(x) \leq w_{x_{o}}^{+}(x)$, for any $x \in \partial \Omega$. Without loss of generality we can assume $x_{o}=0$ and $U\left(x_{o}\right)=0$. Further, we can assume $\Omega \subset \mathbb{R}^{n-1} \times \mathbb{R}_{+}$and that $w_{x_{o}}^{+}$takes the form $w_{x_{o}}^{+}(x)=Q x_{n}$ for some $|Q| \leq Q_{o}$. Now, consider $x \equiv\left(x^{\prime}, x_{n}\right) \in \Omega$ and denote by $\left(x^{\prime}, y_{n}\right) \in \partial \Omega$ the unique point in the boundary $\partial \Omega$ with $0 \leq y_{n}<x_{n}$. Then, we have

$$
\begin{aligned}
u(x) & \leq u\left(x^{\prime}, x_{n}\right)-U\left(x^{\prime}, y_{n}\right)+w_{x_{o}}^{+}\left(x^{\prime}, y_{n}\right) \\
& \leq Q_{1}\left(x_{n}-y_{n}\right)+Q y_{n} \\
& \leq Q_{1}\left(x_{n}-y_{n}\right)+Q_{o} y_{n} \\
& \leq \max \left\{Q_{o}, Q_{1}\right\} x_{n} .
\end{aligned}
$$

We now define the affine function $\widetilde{w}_{x_{o}}^{+}(x):=\max \left\{Q_{o}, Q_{1}\right\} x_{n}$. The previous estimate implies that $u(x) \leq \widetilde{w}_{x_{o}}^{+}(x)$ for any $x \in \Omega$. Moreover, we have $U\left(x_{o}\right)=0=\widetilde{w}_{x_{o}}^{+}\left(x_{o}\right)$ and $u \leq w_{x_{o}}^{+} \leq \widetilde{w}_{x_{o}}^{+}$on $\partial \Omega$, i.e. $U$ satisfies the bounded slope condition for the larger constant $\max \left\{Q_{o}, Q_{1}\right\}$.

\subsection{Mollification in time}

In the definition of variational solutions we are not going to assume any condition on their derivative with respect to time. Therefore, in general we are not allowed to use them as comparison maps in the variational inequality (1.3) and a suitable mollification procedure in time is thus needed. To this end, for $X$ a separable Banach space, an initial datum $v_{o} \in X$ and $1 \leq r \leq \infty$, let $v \in L^{r}(0, T ; X)$ and define the mollification in time of $v$ for $h \in(0, T]$ and $t \in[0, T]$ by means of

$$
[v]_{h}(t):=\mathrm{e}^{-\frac{t}{h}} v_{o}+\frac{1}{h} \int_{0}^{t} \mathrm{e}^{\frac{s-t}{h}} v(s) \mathrm{d} s .
$$

In the applications we are going to use for instance $X=L^{r}\left(\Omega, \mathbb{R}^{N}\right)$ and the related parabolic space $L^{r}(0, T$; $\left.L^{r}\left(\Omega, \mathbb{R}^{N}\right)\right)$. One of the features of the mollification in time is that $[v]_{h}$ solves the ordinary differential equation

$$
\partial_{t}[v]_{h}=\frac{1}{h}\left(v-[v]_{h}\right)
$$

with initial condition $[v]_{h}(0)=v_{o}$. Note that, since $[v]_{h}$ solves the ordinary differential equation (2.2) above, then clearly any common membership of both $v$ and its regularization $[v]_{h}$ to a Banach space is passed also to the time derivative of $[v]_{h}$. This fact will be exploited in $\$ 5.3$ in deriving the uniform a priori bounds for the sequence of $F_{\varepsilon}$-minimizers $u_{\varepsilon}$ which later on imply in particular that the variational solution $u$ possesses a time derivative in $L^{2}\left(\Omega_{T}\right)$.

The basic properties of the mollification in time are summarized in the following lemma (cf. [19, Lemma 2.2] and [3, Appendix B] for the proofs).

Lemma 2.4. Suppose $X$ is a separable Banach space and $v_{o} \in X$. If $v \in L^{r}(0, T ; X)$ for some $r \geq 1$, then also $[v]_{h} \in L^{r}(0, T ; X)$, and $[v]_{h} \rightarrow v$ in $L^{r}(0, T ; X)$ as $h \downarrow 0$ if $r<\infty$. Further, for any $t_{o} \in(0, T]$ there holds

$$
\left\|[v]_{h}\right\|_{L^{r}\left(0, t_{o} ; X\right)} \leq\|v\|_{L^{r}\left(0, t_{o} ; X\right)}+\left[\frac{h}{r}\left(1-\mathrm{e}^{-\frac{t_{o} r}{h}}\right)\right]^{\frac{1}{r}}\left\|v_{o}\right\|_{X} .
$$


In the case $r=\infty$, the bracket $[\ldots]^{1 / r}$ in the preceding inequality has to be interpreted as 1. Moreover, $\partial_{t}[v]_{h} \in$ $L^{r}(0, T ; X)$ with

$$
\partial_{t}[v]_{h}=\frac{1}{h}\left(v-[v]_{h}\right) .
$$

If additionally also $\partial_{t} v \in L^{r}(0, T ; X)$, then

$$
\partial_{t}[v]_{h}=\frac{1}{h} \int_{0}^{t} \mathrm{e}^{\frac{s-t}{h}} \partial_{s} v(s) \mathrm{d} s
$$

and

$$
\left\|\partial_{t}[v]_{h}\right\|_{L^{p}(0, T ; X)} \leq\left\|\partial_{t} v\right\|_{L^{p}(0, T ; X)} .
$$

Finally, if $v \in C^{0}([0, T] ; X)$, then also $[v]_{h} \in C^{0}([0, T] ; X),[v]_{h}(0)=v_{o}$ and $[v]_{h} \rightarrow v$ in $L^{\infty}([0, T] ; X)$ as $h \downarrow 0$.

In the following we want to show that the time mollification of the $f$-energy satisfies $f\left([v]_{h}\right) \leq[f(v)]_{h}$ on $[0, T]$ and $[f(v)]_{h} \rightarrow f(v)$ in $L^{1}(0, T)$ as $h \downarrow 0$ if $v$ and $v_{o}$ are chosen properly. The first estimate will be used frequently throughout the paper, while the convergence is only needed to show that the variational solution $u$ is also a variational solution on any subcylinder $\Omega_{\tau} \subset \Omega_{T}$.

Lemma 2.5. Let $T>0$ and $f: \mathbb{R}^{n} \rightarrow \mathbb{R}$ be convex. Suppose further that $v \in K\left(\Omega_{T}\right)$ and $v_{o} \in W^{1, \infty}(\Omega)$. Then, $[v]_{h} \in K\left(\Omega_{T}\right)$ with

$$
\begin{gathered}
\left\|D[v]_{h}\right\|_{L^{\infty}} \leq \max \left\{\left\|D v_{o}\right\|_{L^{\infty}},\|D v\|_{L^{\infty}}\right\}, \\
f\left(D[v]_{h}\right) \in L^{1}\left(\Omega_{T}\right) \text { and } \\
f\left(D[v]_{h}\right) \leq[f(D v)]_{h} \quad \text { on } \Omega_{T} .
\end{gathered}
$$

Moreover, in the limit $h \downarrow 0$ we have

$$
f\left(D[v]_{h}\right) \rightarrow f(D v) \text { in } L^{1}\left(\Omega_{T}\right) .
$$

Here, $[v]_{h}$ and $[f(D v)]_{h}$ are defined according to (2.1) with $v_{o}$ and $f\left(D v_{o}\right)$ as initial datum.

Proof. The first assertion is a direct consequence of the definition of the mollification, since for $t \in(0, T)$ we have

$$
\begin{aligned}
\left\|D[v]_{h}(t)\right\|_{L^{\infty}} & \leq e^{-\frac{t}{h}}\left\|D v_{o}\right\|_{L^{\infty}}+\frac{1}{h} \int_{0}^{t} e^{\frac{s-t}{h}}\|D v(s)\|_{L^{\infty}} \mathrm{d} s \\
& \leq \max \left\{\left\|D v_{o}\right\|_{L^{\infty}},\|D v\|_{L^{\infty}}\right\}=: R .
\end{aligned}
$$

In turn, this implies $f\left(D[v]_{h}\right) \in L^{1}\left(\Omega_{T}\right)$. Observing that

$$
\frac{1}{h\left(1-\mathrm{e}^{-\frac{t}{h}}\right)} \int_{0}^{t} \mathrm{e}^{\frac{s-t}{h}} \mathrm{~d} s \equiv 1,
$$

usage of the convexity of $f$ gives the estimate

$$
\begin{aligned}
f\left(D[v]_{h}\right) & \leq \mathrm{e}^{-\frac{t}{h}} f\left(D v_{o}\right)+\left(1+\mathrm{e}^{-\frac{t}{h}}\right) f\left(\frac{1}{h\left(1+\mathrm{e}^{-\frac{t}{h}}\right)} \int_{0}^{t} \mathrm{e}^{\frac{s-t}{h}} D v(s) \mathrm{d} s\right) \\
& \leq \mathrm{e}^{-\frac{t}{h}} f\left(D v_{o}\right)+\frac{1}{h} \int_{0}^{t} \mathrm{e}^{\frac{s-t}{h}} f(D v(s)) \mathrm{d} s \\
& =[f(D v)]_{h} .
\end{aligned}
$$


Since $D[v]_{h}$ is strongly convergent in $L^{q}\left(\Omega_{T}, \mathbb{R}^{N}\right)$ for any $q \in[1, \infty)$ to $D v$ as $h \downarrow 0$, we can extract a not relabeled subsequence such that $D[v]_{h} \rightarrow D v$ pointwise almost everywhere on $\Omega_{T}$. Therefore, the continuity of $f$ implies the pointwise almost everywhere convergence $f\left(D[v]_{h}\right) \rightarrow f(D v)$. Taking into account that $\left|f\left(D[v]_{h}\right)\right| \leq \sup _{B_{R}(0)}|f|$, the dominated convergence theorem implies the strong convergence $f\left(D[v]_{h}\right) \rightarrow f(D v)$ in $L^{1}\left(\Omega_{T}\right)$ for the chosen subsequence. Note that the limit does not depend on the subsequence. Therefore, we can repeat the argument starting with an arbitrary subsequence, proving that the convergence holds for the whole sequence.

\section{Gradient constrained obstacle problems}

In this section we consider variational solutions satisfying a gradient constraint of the type $|D u| \leq L$ on $\Omega_{T}$ for some constant $L>0$. This can be achieved in a fairly general setting, in particular without assuming the bounded slope condition. Therefore, we will work with weaker assumptions throughout this section. We consider a bounded open set $\Omega \subset \mathbb{R}^{n}$, a convex integrand $f: \mathbb{R}^{n} \rightarrow \mathbb{R}$ and some initial datum $u_{o}$ satisfying $u_{o} \in W^{1, \infty}(\Omega)$.

Definition 3.1 (Variational solutions, gradient constraint). Let $L>0$ and $u_{o} \in W^{1, \infty}(\Omega)$ such that $L \geq$ $\left\|D u_{o}\right\|_{L^{\infty}\left(\Omega, \mathbb{R}^{n}\right)}$. A map $u \in K_{u_{o}}^{(L)}\left(\Omega_{T}\right)$ with $T \in(0, \infty)$ is called variational solution (of the gradient constrained obstacle problem) in $K_{u_{o}}^{(L)}\left(\Omega_{T}\right)$ if and only if the variational inequality

$$
\begin{aligned}
\iint_{\Omega_{T}} f(D u) \mathrm{d} x \mathrm{~d} t \leq & \iint_{\Omega_{T}}\left[\partial_{t} v(v-u)+f(D v)\right] \mathrm{d} x \mathrm{~d} t \\
& +\frac{1}{2}\left\|v(0)-u_{o}\right\|_{L^{2}(\Omega)}^{2}-\frac{1}{2}\|(v-u)(T)\|_{L^{2}(\Omega)}^{2}
\end{aligned}
$$

holds true for any $v \in K_{u_{o}}^{(L)}\left(\Omega_{T}\right)$ with $\partial_{t} v \in L^{2}\left(\Omega_{T}\right)$. Furthermore, if $T=\infty$, a measurable map $u: \Omega_{\infty} \rightarrow \mathbb{R}$ is termed global variational solution of the gradient constrained obstacle problem if and only if its restriction to any finite cylinder $\Omega_{T}$ with $T \in(0, \infty)$ is a variational solution in $K_{u_{o}}^{(L)}\left(\Omega_{T}\right)$.

Observe that the time independent extension of $u_{o}$ to $\Omega_{T}$, i.e. the map $\bar{u}_{o}(x, t):=u_{o}(x)$ for $(x, t) \in \Omega_{T}$, belongs to the class $K_{u_{o}}^{(L)}\left(\Omega_{T}\right)$ and satisfies $\partial_{t} \bar{u}_{o} \equiv 0$. Hence, the class of admissible testing functions in (3.1) is non-empty. In the sequel we establish in a first step, that variational solutions of the gradient constrained problem in the sense of the preceding definition on the cylinder $\Omega_{T}$ with $T \in(0, \infty)$, also solve the variational inequality (3.1) on any smaller subcylinder $\Omega_{\tau} \subset \Omega_{T}$ with $\tau \in(0, T)$. Note that the following lemmata also apply to variational solutions of the unconstrained problem in the sense of Definition 1.1. The precise statement is as follows:

Lemma 3.2 (Localization in time). Let $L \in(0, \infty], \Omega \subset \mathbb{R}^{n}$ be a bounded, open set, $f: \mathbb{R}^{n} \rightarrow \mathbb{R}$ a convex integrand and $u_{o} \in W^{1, \infty}(\Omega)$ with $\left\|D u_{o}\right\|_{L^{\infty}\left(\Omega, \mathbb{R}^{n}\right)} \leq L$, if $L \in \mathbb{R}$, or $\left\|D u_{o}\right\|_{L^{\infty}\left(\Omega, \mathbb{R}^{n}\right)}<\infty$, if $L=\infty$, respectively. Suppose that $u \in K_{u_{o}}^{(L)}\left(\Omega_{T}\right)$ is a variational solution in $K_{u_{o}}^{(L)}\left(\Omega_{T}\right)$ in the sense of Definition 3.1 (respectively Definition 1.1 in the case $L=\infty)$ on some cylinder $\Omega_{T}$ with $T \in(0, \infty)$. Then, $u$ is also a variational solution in $K_{u_{o}}^{(L)}\left(\Omega_{\tau}\right)$ on any smaller cylinder $\Omega_{\tau}$ with $\tau \in(0, T)$.

Proof. For $\theta \in(0, \tau)$ we consider the cut-off function

$$
\xi_{\theta}(t):=\chi_{[0, \tau-\theta]}(t)+\frac{\tau-t}{\theta} \chi_{(\tau-\theta, \tau]}(t) .
$$

For $v \in K_{u_{o}}^{(L)}\left(\Omega_{\tau}\right)$ satisfying $\partial_{t} v \in L^{2}\left(\Omega_{\tau}\right)$ we define a function $v_{\theta}: \Omega_{T} \rightarrow \mathbb{R}$ by

$$
v_{\theta}:=\xi_{\theta} v+\left(1-\xi_{\theta}\right)[u]_{h},
$$

where $[u]_{h}$ is defined according to (2.1) with $u_{o}$ and $u$ instead of $v_{o}$ and $v$, respectively and $\xi_{\theta} v$ has been extended from $\Omega_{\tau}$ to $\Omega_{T}$ by 0 . Since $v_{\theta} \in K_{u_{o}}^{(L)}\left(\Omega_{T}\right)$ and $\partial_{t} v_{\theta} \in L^{2}\left(\Omega_{T}\right)$ we are allowed to choose $v_{\theta}$ as comparison map in (3.1). This yields 


$$
\begin{aligned}
\iint_{\Omega_{T}} f(D u) \mathrm{d} x \mathrm{~d} t \leq & \iint_{\Omega_{T}}\left[\partial_{t} v_{\theta}\left(v_{\theta}-u\right)+f\left(D v_{\theta}\right)\right] \mathrm{d} x \mathrm{~d} t \\
& +\frac{1}{2}\left\|v(0)-u_{o}\right\|_{L^{2}}^{2}-\frac{1}{2}\left\|\left([u]_{h}-u\right)(T)\right\|_{L^{2}}^{2} .
\end{aligned}
$$

In the following we want to pass to the limit $\theta \downarrow 0$. Therefore, we have a look at the first two terms on the right-hand side of the previous inequality. The first one can be rewritten to

$$
\begin{aligned}
& \iint_{\Omega_{T}} \partial_{t} v_{\theta}\left(v_{\theta}-u\right) \mathrm{d} x \mathrm{~d} t \\
& =\iint_{\Omega \times(0, \tau-\theta)} \partial_{t} v(v-u) \mathrm{d} x \mathrm{~d} t+\iint_{\Omega \times(\tau, T)} \partial_{t}[u]_{h}\left([u]_{h}-u\right) \mathrm{d} x \mathrm{~d} t \\
& \quad+\iiint_{\Omega \times(\tau-\theta, \tau)} \xi_{\theta}^{\prime} \xi_{\theta}\left|v-[u]_{h}\right|^{2} \mathrm{~d} x \mathrm{~d} t+\iint_{\Omega \times(\tau-\theta, \tau)} \xi_{\theta}^{\prime}\left([u]_{h}-u\right)\left(v-[u]_{h}\right) \mathrm{d} x \mathrm{~d} t \\
& \quad+\iint_{\Omega \times(\tau-\theta, \tau)}\left[\xi_{\theta} \partial_{t} v+\left(1-\xi_{\theta}\right) \partial_{t}[u]_{h}\right]\left[\xi_{\theta}(v-u)+\left(1-\xi_{\theta}\right)\left([u]_{h}-u\right)\right] \mathrm{d} x \mathrm{~d} t \\
& =: \mathbf{I}_{\theta}+\mathbf{I I}_{\theta}+\mathbf{I I I}_{\theta}+\mathbf{I V}_{\theta}+\mathbf{V}_{\theta},
\end{aligned}
$$

where the meaning of the terms $\mathbf{I}_{\theta}-\mathbf{V}_{\theta}$ is obvious in this context. Note that the properties of the appearing functions imply

$$
\lim _{\theta \downarrow 0} \mathbf{I}_{\theta}=\iint_{\Omega \times(0, \tau)} \partial_{t} v(v-u) \mathrm{d} x \mathrm{~d} t, \quad \lim _{\theta \downarrow 0} \mathbf{I I I}_{\theta}=-\frac{1}{2}\left\|\left(v-[u]_{h}\right)(\tau)\right\|_{L^{2}}^{2},
$$

and

$$
\lim _{\theta \downarrow 0} \mathbf{V}_{\theta}=0, \quad \limsup _{\theta \downarrow 0} \mathbf{I} \mathbf{V}_{\theta} \leq\left\|\left([u]_{h}-u\right)\left(v-[u]_{h}\right)(\tau)\right\|_{L^{1}},
$$

so that

$$
\begin{aligned}
\underset{\theta \downarrow 0}{\limsup } \iint_{\Omega_{T}} \partial_{t} v_{\theta}\left(v_{\theta}-u\right) \mathrm{d} x \mathrm{~d} t \\
\leq \quad \iint_{\Omega \times(0, \tau)} \partial_{t} v(v-u) \mathrm{d} x \mathrm{~d} t+\iint_{\Omega \times(\tau, T)} \partial_{t}[u]_{h}\left([u]_{h}-u\right) \mathrm{d} x \mathrm{~d} t \\
\quad-\frac{1}{2}\left\|\left(v-[u]_{h}\right)(\tau)\right\|_{L^{2}}^{2}+\left\|\left([u]_{h}-u\right)\left(v-[u]_{h}\right)(\tau)\right\|_{L^{1}} .
\end{aligned}
$$

The second term appearing on the right-hand side of the minimality condition (3.2) is given by

$$
\begin{aligned}
\iint_{\Omega_{T}} f\left(D v_{\theta}\right) \mathrm{d} x \mathrm{~d} t= & \iint_{\Omega \times(\tau-\theta, \tau)} f\left(\xi_{\theta} D v+\left(1-\xi_{\theta}\right) D[u]_{h}\right) \mathrm{d} x \mathrm{~d} t \\
& +\iiint_{\Omega \times(0, \tau-\theta)} f(D v) \mathrm{d} x \mathrm{~d} t+\iint_{\Omega \times(\tau, T)} f\left(D[u]_{h}\right) \mathrm{d} x \mathrm{~d} t .
\end{aligned}
$$

Using the convexity of $f$, it is clear that the second term of the previous equality tends to 0 as $\theta \downarrow 0$, so that

$$
\lim _{\theta \downarrow 0} \iint_{\Omega_{T}} f\left(D v_{\theta}\right) \mathrm{d} x \mathrm{~d} t=\iint_{\Omega \times(0, \tau)} f(D v) \mathrm{d} x \mathrm{~d} t+\iint_{\Omega \times(\tau, T)} f\left(D[u]_{h}\right) \mathrm{d} x \mathrm{~d} t .
$$

Therefore, after passing to the limit $\theta \downarrow 0$, the variational inequality (3.2) implies 


$$
\begin{aligned}
\iint_{\Omega_{\tau}} f(D u) \mathrm{d} x \mathrm{~d} t \leq & \iint_{\Omega_{\tau}}\left[\partial_{t} v(v-u)+f(D v)\right] \mathrm{d} x \mathrm{~d} t \\
& +\frac{1}{2}\left\|v(0)-u_{o}\right\|_{L^{2}}^{2}-\frac{1}{2}\left\|\left(v-[u]_{h}\right)(\tau)\right\|_{L^{2}}^{2} \\
& +\iiint_{\Omega \times(\tau, T)}\left[\partial_{t}[u]_{h}\left([u]_{h}-u\right) \mathrm{d} x+f\left(D[u]_{h}\right)-f(D u)\right] \mathrm{d} x \mathrm{~d} t \\
& -\frac{1}{2}\left\|\left([u]_{h}-u\right)(T)\right\|_{L^{2}}^{2}+\left\|\left([u]_{h}-u\right)\left(v-[u]_{h}\right)(\tau)\right\|_{L^{1}} .
\end{aligned}
$$

From Lemmas 2.4 and 2.5 we know that $\partial_{t}[u]_{h}\left([u]_{h}-u\right) \leq 0$ and the convergences $[u]_{h} \rightarrow u$ in $L^{\infty}\left(0, T ; L^{2}\left(\Omega, \mathbb{R}^{N}\right)\right)$ and $f\left(D[u]_{h}\right) \rightarrow f(D u)$ in $L^{1}\left(\Omega_{T}\right)$ as $h \downarrow 0$. Therefore, passing to the limit $h \downarrow 0$ in the last inequality, we conclude that $u$ satisfies the variational inequality (1.3) on the subcylinder $\Omega_{\tau}$. Since $v$ was arbitrary, $u$ is therefore a variational solution on the subcylinder $\Omega_{\tau}$.

As application of the localization in time principle from Lemma 3.2 we establish that variational solutions fulfill the initial condition $u(0)=u_{o}$ in the strong $L^{2}(\Omega)$-sense. The precise statement is as follows:

Lemma 3.3. Let $L \in(0, \infty], \Omega \subset \mathbb{R}^{n}$ a bounded open set, $f: \mathbb{R}^{n} \rightarrow \mathbb{R}$ a convex integrand and an initial datum $u_{o} \in W^{1, \infty}(\Omega)$ with $\left\|D u_{o}\right\|_{L^{\infty}\left(\Omega, \mathbb{R}^{n}\right)} \leq L$, if $L \in \mathbb{R}$, or $\left\|D u_{o}\right\|_{L^{\infty}\left(\Omega, \mathbb{R}^{n}\right)}<\infty$, if $L=\infty$, respectively. Then, any variational solution $u$ in $K_{u_{o}}^{(L)}\left(\Omega_{T}\right)$ in the sense of Definition 3.1 (respectively Definition 1.1 if $L=\infty$ ) on some cylinder $\Omega_{T}$ with $T \in(0, \infty]$ satisfies

$$
\lim _{t \downarrow 0}\left\|u(t)-u_{o}\right\|_{L^{2}(\Omega)}^{2}=0 .
$$

Proof. From Lemma 3.2 we know that $u$ fulfills the variational inequality (3.1) on any subcylinder $\Omega_{\tau}$ for $\tau \in(0, T)$. Moreover, for the time-independent extension $\bar{u}_{o}(x, t):=u_{o}(x)$ of $u_{o}$ we have that $\bar{u}_{o} \in K_{u_{o}}^{(L)}\left(\Omega_{\tau}\right)$ and $\partial_{t} \bar{u}_{o} \in L^{2}\left(\Omega_{\tau}\right)$. Therefore, choosing $v=\bar{u}_{o}$ as comparison function in (3.1) yields

$$
\iint_{\Omega_{\tau}} f(D u) \mathrm{d} x \mathrm{~d} t+\frac{1}{2}\left\|u(\tau)-u_{o}\right\|_{L^{2}(\Omega)}^{2} \leq \iint_{\Omega_{\tau}} f\left(D u_{o}\right) \mathrm{d} x \mathrm{~d} t=\tau \int_{\Omega} f\left(D u_{o}\right) \mathrm{d} x<\infty .
$$

Since $M:=\max \left\{\|D u\|_{L^{\infty}\left(\Omega_{\tau}\right)},\left\|D u_{o}\right\|_{L^{\infty}(\Omega)}\right\}<\infty$ (note in the case $L<\infty$ we have $M \leq L$ ), this implies

$$
\left\|u(\tau)-u_{o}\right\|_{L^{2}(\Omega}^{2} \leq 4 \tau|\Omega| \sup _{B_{M}(0)}|f| \quad \forall \tau \in(0, T) .
$$

This proves the claim of the lemma.

Next, we will establish that any variational solution $u$ to the constrained problem admits a time derivative $\partial_{t} u$ in a weak sense and that it belongs to $L^{2}\left(\Omega_{T}\right)$. Note, since the case $L=\infty$ is included in Lemma 3.4, the first assertion in Theorem 1.3 follows as a byproduct.

Lemma 3.4. Let $L \in(0, \infty], \Omega$ a bounded open subset of $\mathbb{R}^{n}, f: \mathbb{R}^{n} \rightarrow \mathbb{R}$ a convex integrand and an initial datum $u_{o} \in W^{1, \infty}(\Omega)$ with $\left\|D u_{o}\right\|_{L^{\infty}\left(\Omega, \mathbb{R}^{n}\right)} \leq L$, if $L \in \mathbb{R}$, or $\left\|D u_{o}\right\|_{L^{\infty}\left(\Omega, \mathbb{R}^{n}\right)}<\infty$, if $L=\infty$, respectively. Suppose that $u$ is a variational solution in $K_{u_{o}}^{(L)}\left(\Omega_{T}\right)$ in the sense of Definition 3.1 or Definition 1.1 in the case $L=\infty$ on some cylinder $\Omega_{T}$ with $T \in(0, \infty]$. Then, we have $\partial_{t} u \in L^{2}\left(\Omega_{T}\right)$ together with the estimate

$$
\iint_{\Omega_{T}}\left|\partial_{t} u\right|^{2} \mathrm{~d} x \mathrm{~d} t \leq 2|\Omega| \sup _{B_{M}(0)} f
$$

where $M \geq \max \left\{\|D u\|_{L^{\infty}\left(\Omega_{T}\right)},\left\|D u_{o}\right\|_{L^{\infty}(\Omega)}\right\}$. In particular, if $L<\infty$ the previous estimate holds with $M=L$. 
Proof. From Lemma 3.2 we know that $u$ is also a variational solution on any subcylinder $\Omega_{\tau}$ for $\tau \in \mathbb{R} \cap(0, T]$. We define $[u]_{h}$ according to (2.1) with $\left(v_{o}, v\right)$ replaced by $\left(u_{o}, u\right)$. Then, $[u]_{h} \in K_{u_{o}}^{(L)}\left(\Omega_{\tau}\right)$ and $\partial_{t}[u]_{h} \in L^{2}\left(\Omega_{\tau}\right)$. Therefore, we are allowed to test the variational inequality (3.1) on $\Omega_{\tau}$ with the comparison map $v=[u]_{h}$. Using also Lemma 2.5 and the fact that $\frac{1}{2}\left\|\left([u]_{h}-u\right)(\tau)\right\|_{L^{2}(\Omega)}^{2} \geq 0$, this implies

$$
\begin{aligned}
-\iint_{\Omega_{\tau}} \partial_{t}[u]_{h}\left([u]_{h}-u\right) \mathrm{d} x \mathrm{~d} t & \leq \iint_{\Omega_{\tau}}\left[f\left(D[u]_{h}\right)-f(D u)\right] \mathrm{d} x \mathrm{~d} t \\
& \leq \iint_{\Omega_{\tau}}\left[[f(D u)]_{h}-f(D u)\right] \mathrm{d} x \mathrm{~d} t \\
& =-h \iint_{\Omega_{\tau}} \partial_{t}[f(D u)]_{h} \mathrm{~d} x \mathrm{~d} t \\
& =h\left[\int_{\Omega} f\left(D u_{o}\right) \mathrm{d} x-\int_{\Omega \times\{\tau\}}[f(D u)]_{h} \mathrm{~d} x\right],
\end{aligned}
$$

where $[f(D u)]_{h}$ is defined according to (2.1) with $v_{o}$ and $v$ replaced by $f\left(D u_{o}\right)$ and $f(D u)$, respectively. We now choose $M \geq \max \left\{\|D u\|_{L^{\infty}\left(\Omega_{T}\right)},\left\|D u_{o}\right\|_{L^{\infty}(\Omega)}\right\}$. Observe that in the case $L<\infty$, the choice $M=L$ is possible. Exploiting the fact that $[u]_{h}-u=-h \partial_{t}[u]_{h}$ by Lemma 2.4, this yields the uniform bound

$$
\iint_{\Omega_{\tau}}\left|\partial_{t}[u]_{h}\right|^{2} \mathrm{~d} x \mathrm{~d} t \leq 2|\Omega| \sup _{B_{M}(0)}|f| \quad \forall h \in(0, \tau],
$$

such that the time derivative $\partial_{t} u$ exists with $\partial_{t} u \in L^{2}\left(\Omega_{\tau}\right)$ for all $\tau \in(0, T]$ together with the quantitative estimate

$$
\iint_{\Omega_{\tau}}\left|\partial_{t} u\right|^{2} \mathrm{~d} x \mathrm{~d} t \leq 2|\Omega| \sup _{B_{M}(0)}|f| \quad \forall \tau \in(0, T] .
$$

Therefore, if $T<\infty$, setting $\tau=T$, or otherwise, if $T=\infty$, letting $\tau \rightarrow \infty$, shows the claim that $\partial_{t} u \in L^{2}\left(\Omega_{T}\right)$ together with the asserted estimate.

\section{Parabolic minimizers and the comparison principle}

Observe, that the assertion of Lemma 3.4, i.e. the fact that variational solutions admit a time derivative $\partial_{t} u \in$ $L^{2}\left(\Omega_{T}\right)$, allows an integration by parts in the first term on the right-hand side of the variational inequality (3.1). The integration by parts shows that

$$
\iint_{\Omega_{T}} f(D u) \mathrm{d} x \mathrm{~d} t \leq \iint_{\Omega_{T}}\left[\partial_{t} u(v-u)+f(D v)\right] \mathrm{d} x \mathrm{~d} t
$$

holds true for any $v \in K_{u_{o}}^{(L)}\left(\Omega_{T}\right)$ with $\partial_{t} v \in L^{2}\left(\Omega_{T}\right)$. This motivates the following definition:

Definition 4.1 (Parabolic minimizer). Let $L \in(0, \infty]$. A map $u \in K^{(L)}\left(\Omega_{T}\right)$ with $T \in(0, \infty)$ and $\partial_{t} u \in L^{2}\left(\Omega_{T}\right)$ is called parabolic minimizer (of the gradient constrained obstacle problem in the case $L<\infty)$ in $K^{(L)}\left(\Omega_{T}\right.$ ) if and only if the variational inequality

$$
\iint_{\Omega_{T}} f(D u) \mathrm{d} x \mathrm{~d} t \leq \iint_{\Omega_{T}}\left[\partial_{t} u(v-u)+f(D v)\right] \mathrm{d} x \mathrm{~d} t
$$

holds true for any $v \in K_{u}^{(L)}\left(\Omega_{T}\right)$. Further, if $T=\infty$, a measurable map $u: \Omega_{\infty} \rightarrow \mathbb{R}$ is termed global parabolic minimizer (of the gradient constraint obstacle problem) if and only if its restriction to any finite cylinder $\Omega_{T}$ with $T \in(0, \infty)$ is a parabolic minimizer in $K^{(L)}\left(\Omega_{T}\right)$. 
We note that the notion of parabolic minimizers to vector valued integrands with quadratic growth has its origin in a paper by Wieser [33].

Remark 4.2 (Localization in space). In the proof of the main Theorem 1.2 it is crucial, that a localization principle with respect to space holds true. By this we mean that any parabolic minimizer of the gradient constrained obstacle problem in $\Omega_{T}$, with $\Omega$ convex, in the sense of Definition 4.1 is also a parabolic minimizer of the gradient constrained problem on any smaller subcylinder $\Omega^{\prime} \times(0, T)$, with $\Omega^{\prime}$ a convex, open subset of $\Omega$. The proof of this basic fact is elementary and could be skipped. For completeness reasons we however include the argument, which is based on the identification of the spaces $W^{1, \infty}(\Omega)$ and $C^{0,1}(\bar{\Omega})$ as explained in Proposition 2.1. Recall that $w \in W^{1, \infty}(\Omega)$ with $\|D w\|_{L^{\infty}\left(\Omega, \mathbb{R}^{n}\right)} \leq L$ has a Lipschitz continuous representative, still denoted by $w$, with $[w]_{0,1 ; \Omega} \leq L$.

Suppose, $L \in(0, \infty)$ and $u \in K^{(L)}\left(\Omega_{T}\right)$ with $\partial_{t} u \in L^{2}\left(\Omega_{T}\right)$ is a parabolic minimizer in the sense of Definition 4.1. Consider $v \in K_{u}^{(L)}\left(\Omega_{T}^{\prime}\right)$. Observe that $v=u$ on the lateral boundary $\partial \Omega^{\prime} \times(0, T)$. Define the comparison $\operatorname{map} \tilde{v}: \Omega_{T} \rightarrow \mathbb{R}$ by

$$
\tilde{v}:=\left\{\begin{array}{l}
v \text { on } \Omega^{\prime} \times(0, T) \\
u \text { on }\left(\Omega \backslash \Omega^{\prime}\right) \times(0, T)
\end{array}\right.
$$

Clearly, this construction provides us with a map $\tilde{v} \in L^{\infty}\left(\Omega_{T}\right) \cap C^{0}\left([0, T] ; L^{2}(\Omega)\right)$ such that $\partial_{t} \tilde{v} \in L^{2}\left(\Omega_{T}\right)$. Further, for a.e. $t \in(0, T)$ we have

$$
|\tilde{v}(x, t)-\tilde{v}(y, t)| \leq L|x-y|
$$

whenever $x, y \in \overline{\Omega^{\prime}}$ or $x, y \in \overline{\Omega \backslash \Omega^{\prime}}$. In the case that $x \in \Omega^{\prime}$ and $y \in \Omega \backslash \Omega^{\prime}$ we find $z \in \partial \Omega^{\prime} \cap[x, y]$, which allows for the estimate (note that $v(z, t)=\tilde{v}(z, t)=u(z, t)$ )

$$
\begin{aligned}
|\tilde{v}(x, t)-\tilde{v}(y, t)| & \leq|\tilde{v}(x, t)-\tilde{v}(z, t)|+|\tilde{v}(z, t)-\tilde{v}(y, t)| \\
& =|v(x, t)-v(z, t)|+|u(z, t)-u(y, t)| \\
& \leq L|x-z|+L|z-y|=L|x-y|,
\end{aligned}
$$

establishing $[\tilde{v}(t)]_{0,1 ; \Omega} \leq L$. Observing that $\tilde{v}=u$ on $\partial \Omega \times(0, T)$, we have shown that $\tilde{v} \in K_{u}^{(L)}\left(\Omega_{T}\right)$ and therefore $\tilde{v}$ is admissible in the minimality condition (4.1). Since $\tilde{v}=u$ on $\left(\Omega \backslash \Omega^{\prime}\right) \times(0, T)$, the minimality condition can be re-written as

$$
\iint_{\Omega_{T}^{\prime}} f(D u) \mathrm{d} x \mathrm{~d} t \leq \iint_{\Omega_{T}^{\prime}}\left[\partial_{t} u(v-u)+f(D v)\right] \mathrm{d} x \mathrm{~d} t,
$$

proving that $u$ is a parabolic minimizer in the sense of Definition 4.1 on the subcylinder $\Omega_{T}^{\prime}$.

The localization principle in space will be applied to parabolic minimizers $u$ on $\Omega_{T}$ with a bounded, convex open set $\Omega$ and its translate $u_{y}(x, t):=u(x+y, t)$ on $\Omega_{y} \times(0, T)$ with $\Omega_{y}:=\left\{x-y \in \mathbb{R}^{n}: x \in \Omega\right\}$ on the intersection $\left(\Omega \cap \Omega_{y}\right) \times(0, T)$. Observe, that the convexity assumptions are clearly fulfilled in this special case.

In the next lemma we are concerned with the comparison principle for parabolic minimizers.

Lemma 4.3 (Comparison principle). Let $L \in(0, \infty]$ and suppose that $\Omega \subset \mathbb{R}^{n}$ is a bounded open set, $f: \mathbb{R}^{n} \rightarrow \mathbb{R}$ a convex integrand and $u, \tilde{u} \in K^{(L)}\left(\Omega_{T}\right)$ with $\partial_{t} u, \partial_{t} \tilde{u} \in L^{2}\left(\Omega_{T}\right)$ parabolic minimizers in the sense of Definition 4.1 on $\Omega_{T}$ for some $T \in(0, \infty]$. Suppose further that $u \leq \tilde{u}$ on $\partial_{\mathcal{P}} \Omega_{T}$. Then, we have

$$
u \leq \tilde{u} \text { a.e. in } \Omega_{T} .
$$

Proof. Let $\tau \in \mathbb{R} \cap(0, T]$. We define $v:=\min \{u, \tilde{u}\}$ and $w:=\max \{u, \tilde{u}\}$ on $\Omega_{\tau}$ and $v:=u, w:=\tilde{u}$ on $\Omega \times(\tau, T)$. We note that $v \in K_{u}^{(L)}\left(\Omega_{T}\right)$ and $w \in K_{\tilde{u}}^{(L)}\left(\Omega_{T}\right)$. This ensures that $v$ is admissible as comparison function in the variational inequality (3.1) on $\Omega_{\tau}$ for $u$ and $w$ is admissible in the variational inequality for $\tilde{u}$. Adding the two resulting inequalities and using that $v(0)=u_{o}$ and $w(0)=\tilde{u}_{o}$, we obtain 


$$
\begin{aligned}
& \iint_{\Omega_{\tau}}[f(D u)+f(D \tilde{u})] \mathrm{d} x \mathrm{~d} t \\
& \quad \leq \iint_{\Omega_{\tau}}\left[f(D v)+f(D w)+\partial_{t} u(v-u)+\partial_{t} \tilde{u}(w-\tilde{u})\right] \mathrm{d} x \mathrm{~d} t .
\end{aligned}
$$

We now consider the terms on the right-hand side of (4.2). From the definition of $v$ and $w$ we have

$$
\iint_{\Omega_{\tau}}[f(D v)+f(D w)] \mathrm{d} x \mathrm{~d} t=\iint_{\Omega_{\tau}}[f(D u)+f(D \tilde{u})] \mathrm{d} x \mathrm{~d} t .
$$

Again, from the definition of $v$ and $w$, we observe that $v-u=-(u-\tilde{u})_{+}$and $w-\tilde{u}=(u-\tilde{u})_{+}$, so that

$$
\begin{aligned}
\partial_{t} u(v-u)+\partial_{t} \tilde{u}(w-\tilde{u}) & =-\partial_{t}(u-\tilde{u})(u-\tilde{u})_{+} \\
& =-\partial_{t}(u-\tilde{u})_{+}(u-\tilde{u})_{+}=-\frac{1}{2} \partial_{t}(u-\tilde{u})_{+}^{2},
\end{aligned}
$$

implying that

$$
\begin{aligned}
\iint_{\Omega_{\tau}}\left[\partial_{t} u(v-u)+\partial_{t} \tilde{u}(w-\tilde{u})\right] \mathrm{d} x \mathrm{~d} t & =-\frac{1}{2} \iint_{\Omega_{\tau}} \partial_{t}(u-\tilde{u})_{+}^{2} \mathrm{~d} x \mathrm{~d} t \\
& =-\frac{1}{2} \int_{\Omega \times\{\tau\}}(u-\tilde{u})_{+}^{2} \mathrm{~d} x .
\end{aligned}
$$

Joining the preceding estimates with (4.2), we find that

$$
\int_{\Omega \times\{\tau\}}(u-\tilde{u})_{+}^{2} \mathrm{~d} x \leq 0 .
$$

Since $\tau \in \mathbb{R} \cap(0, T]$ was arbitrary, this proves the claim that $u \leq \tilde{u}$ a.e. in $\Omega_{T}$.

The preceding comparison principle can be used to establish the following maximum principle.

Lemma 4.4 (Maximum principle). Let $L \in(0, \infty]$ and suppose that $\Omega \subset \mathbb{R}^{n}$ is open and bounded, $f: \mathbb{R}^{n} \rightarrow \mathbb{R}$ convex and let $u, \tilde{u} \in K^{(L)}\left(\Omega_{T}\right)$ with $\partial_{t} u, \partial_{t} \tilde{u} \in L^{2}\left(\Omega_{T}\right)$ be parabolic minimizers in the sense of Definition 4.1 on $\Omega_{T}$ for some $T \in(0, \infty]$. Then, we have

$$
\sup _{\Omega_{T}}(u-\tilde{u})=\sup _{\partial_{\mathcal{P}} \Omega_{T}}(u-\tilde{u}) .
$$

Proof. For $(x, t) \in \partial_{\mathcal{P}} \Omega_{T}$ we have

$$
u(x, t)=\tilde{u}(x, t)+u(x, t)-\tilde{u}(x, t) \leq \tilde{u}(x, t)+\sup _{\partial \mathcal{P} \Omega_{T}}(u-\tilde{u}) .
$$

Moreover, both $u$ and $\tilde{u}+\sup _{\partial_{\mathcal{P}} \Omega_{T}}(u-\tilde{u})$ are parabolic minimizers on $\Omega_{T}$. Therefore, Lemma 4.3 yields that

$$
u \leq \tilde{u}+\sup _{\partial \mathcal{P} \Omega_{T}}(u-\tilde{u}) \quad \text { on } \Omega_{T},
$$

which is the same as

$$
\sup _{\Omega_{T}}(u-\tilde{u}) \leq \sup _{\partial \mathcal{P} \Omega_{T}}(u-\tilde{u}) .
$$

Since the reversed inequality holds trivially, this proves the claim. 


\section{Elliptic regularization}

In this section we show, how the method of elliptic regularization can be used to establish the existence of a variational solution to the gradient constrained obstacle problem in the sense of Definition 3.1. Let $T \in(0, \infty)$. For $\varepsilon \in(0,1]$ we consider convex variational integrals of the form

$$
F_{\varepsilon}(v):=\iint_{\Omega_{T}} \mathrm{e}^{-\frac{t}{\varepsilon}}\left[\frac{1}{2}\left|\partial_{t} v\right|^{2}+\frac{1}{\varepsilon} f(D v)\right] \mathrm{d} x \mathrm{~d} t .
$$

In the following, for given $u_{o} \in W^{1, \infty}(\Omega)$ with $\left\|D u_{o}\right\|_{L^{\infty}\left(\Omega, \mathbb{R}^{n}\right)} \leq L$ we look for a minimizer of the functional $F_{\varepsilon}$ within the function space

$$
\widehat{K}_{u_{o}}^{(L)}\left(\Omega_{T}\right):=\left\{v \in K_{u_{o}}^{(L)}\left(\Omega_{T}\right): \partial_{t} v \in L^{2}\left(\Omega_{T}\right), v(0)=u_{o}\right\} .
$$

Keep in mind, that functions $v \in \widehat{K}_{u_{o}}^{(L)}\left(\Omega_{T}\right)$ belong to the space $C^{0, \frac{1}{2}}\left([0, T] ; L^{2}(\Omega)\right)$, due to the $L^{2}$-bound $\left\|\partial_{t} v\right\|_{L^{2}\left(\Omega_{T}\right)}<\infty$, and therefore the initial condition $v(0)=u_{o}$ holds in the strong $L^{2}(\Omega)$-sense. Observe that the time independent extension of $u_{o}$ to the cylinder $\Omega_{T}$, i.e. the map $\bar{u}_{o}(x, t):=u_{o}(x)$ for $(x, t) \in \Omega_{T}$, belongs to the class $\widehat{K}_{u_{o}}^{(L)}\left(\Omega_{T}\right)$, so that this class is non-empty. Moreover, observe that

$$
\begin{aligned}
F_{\varepsilon}\left(\bar{u}_{o}\right) & =\int_{\Omega} f\left(D u_{o}\right) \mathrm{d} x \int_{0}^{T} \frac{1}{\varepsilon} \mathrm{e}^{-\frac{t}{\varepsilon}} \mathrm{d} t \\
& =\left(1-\mathrm{e}^{-\frac{T}{\varepsilon}}\right) \int_{\Omega} f\left(D u_{o}\right) \mathrm{d} x \leq\left(1-\mathrm{e}^{-\frac{T}{\varepsilon}}\right)|\Omega| \sup _{B_{L}(0)}|f| .
\end{aligned}
$$

Furthermore, mappings $v \in \widehat{K}_{u_{o}}^{(L)}\left(\Omega_{T}\right)$ admit the following bound on the time derivative (note that $\|D v\|_{L^{\infty}\left(\Omega_{T}, \mathbb{R}^{n}\right)} \leq$ $L)$ :

$$
\begin{aligned}
\mathrm{e}^{-\frac{T}{\varepsilon}} \iint_{\Omega_{T}} \frac{1}{2}\left|\partial_{t} v\right|^{2} \mathrm{~d} x \mathrm{~d} t & \leq F_{\varepsilon}(v)-\frac{1}{\varepsilon} \iint_{\Omega_{T}} \mathrm{e}^{-\frac{t}{\varepsilon}} f(D v) \mathrm{d} x \mathrm{~d} t \\
& \leq F_{\varepsilon}(v)+\left(1-\mathrm{e}^{-\frac{T}{\varepsilon}}\right)|\Omega| \sup _{B_{L}(0)}|f| .
\end{aligned}
$$

Taking an arbitrary point $x_{o} \in \partial \Omega$, we have for a.e. $t \in(0, T)$ and $x \in \bar{\Omega}$ that

$$
|v(x, t)| \leq\left|v(x, t)-v\left(x_{o}, t\right)\right|+\left|u_{o}\left(x_{o}\right)\right| \leq\left\|u_{o}\right\|_{L^{\infty}(\Omega)}+L \operatorname{diam}(\Omega),
$$

so that

$$
\|v\|_{L^{\infty}\left(\Omega_{T}\right)}+\|D v\|_{L^{\infty}\left(\Omega_{T}, \mathbb{R}^{n}\right)} \leq\left\|u_{o}\right\|_{L^{\infty}(\Omega)}+L(1+\operatorname{diam}(\Omega))
$$

holds true for any $v \in \widehat{K}_{u_{o}}^{(L)}\left(\Omega_{T}\right)$. Finally, for any $0 \leq t_{1}<t_{2} \leq T$ we have

$$
\left\|v\left(t_{2}\right)-v\left(t_{1}\right)\right\|_{L^{2}(\Omega)}^{2} \leq\left(t_{2}-t_{1}\right)\left\|\partial_{t} v\right\|_{L^{2}\left(\Omega_{T}\right)}^{2},
$$

which together with (5.2) yields that $v$ is a $\frac{1}{2}$-Hölder-continuous map from $[0, T]$ to $L^{2}(\Omega)$, i.e. $v \in C^{0, \frac{1}{2}}([0, T]$; $\left.L^{2}(\Omega)\right)$ holds true for any $v \in \widehat{K}_{u_{o}}^{(L)}\left(\Omega_{T}\right)$.

\subsection{Existence of approximations}

In this section we establish the existence of minimizers to the energy functionals $F_{\varepsilon}$.

Lemma 5.1. For any $\varepsilon \in(0,1]$, the functional $F_{\varepsilon}$ admits a unique minimizer $u_{\varepsilon}$ in the class $\widehat{K}_{u_{o}}^{(L)}\left(\Omega_{T}\right)$. 
Proof. From (5.1) we obtain the lower bound

$$
F_{\varepsilon}(v) \geq-\left(1-\mathrm{e}^{-\frac{T}{\varepsilon}}\right)|\Omega| \sup _{B_{L}(0)}|f|,
$$

for the $F_{\varepsilon}$-energy of maps $v \in \widehat{K}_{u_{o}}^{(L)}\left(\Omega_{T}\right)$. We now consider a minimizing sequence $u_{j} \in \widehat{K}_{u_{o}}^{(L)}\left(\Omega_{T}\right), j \in \mathbb{N}$, i.e.

$$
\lim _{j \rightarrow \infty} F_{\varepsilon}\left(u_{j}\right)=\inf _{v \in \widehat{K}_{u_{o}}^{(L)}\left(\Omega_{T}\right)} F_{\varepsilon}(v) \leq F_{\varepsilon}\left(\bar{u}_{o}\right) \leq\left(1-\mathrm{e}^{-\frac{T}{\varepsilon}}\right)|\Omega| \sup _{B_{L}(0)}|f| .
$$

Hence, we can assume without loss of generality that $F_{\varepsilon}\left(u_{j}\right) \leq|\Omega| \sup _{B_{L}(0)}|f|$ for any $j \in \mathbb{N}$. Applying (5.1) to the minimizing sequence $u_{j}$ we find that

$$
\mathrm{e}^{-\frac{T}{\varepsilon}} \iint_{\Omega_{T}} \frac{1}{2}\left|\partial_{t} u_{j}\right|^{2} \mathrm{~d} x \mathrm{~d} t \leq\left(2-\mathrm{e}^{-\frac{T}{\varepsilon}}\right)|\Omega| \sup _{B_{L}(0)}|f|
$$

holds true for any $j \in \mathbb{N}$. The preceding inequality immediately implies the following uniform bound for the time derivative:

$$
\sup _{j \in \mathbb{N}} \iint_{\Omega_{T}}\left|\partial_{t} u_{j}\right|^{2} \mathrm{~d} x \mathrm{~d} t \leq 4 \mathrm{e}^{\frac{T}{\varepsilon}}|\Omega| \sup _{B_{L}(0)}|f| .
$$

From (5.2) we derive the uniform $L^{\infty}-W^{1, \infty}$ bound

$$
\sup _{j \in \mathbb{N}}\left[\left\|u_{j}\right\|_{L^{\infty}\left(\Omega_{T}\right)}+\left\|D u_{j}\right\|_{L^{\infty}\left(\Omega_{T}\right)}\right] \leq\left\|u_{o}\right\|_{L^{\infty}(\Omega)}+L(1+\operatorname{diam}(\Omega)) .
$$

Thus, there exists a map $u_{\varepsilon}$, which we will denote for simplicity by $u$ throughout the proof, satisfying

$$
u \in \bigcap_{q \geq 1} L^{q}\left(0, T ; W^{1, p}(\Omega)\right) \quad \text { with } \quad \partial_{t} u \in L^{2}\left(\Omega_{T}\right)
$$

and a subsequence of $\left(u_{j}\right)_{j \in \mathbb{N}}$ (still denoted this way) such that

$$
\left\{\begin{array}{c}
u_{j} \rightarrow u \quad \text { weakly in } L^{q}\left(\Omega_{T}\right) \text { for any } q \geq 1, \\
D u_{j} \rightarrow D u \text { weakly in } L^{q}\left(\Omega_{T}, \mathbb{R}^{n}\right) \text { for any } q \geq 1, \\
\partial_{t} u_{j} \rightarrow \partial_{t} u \text { weakly in } L^{2}\left(\Omega_{T}\right) .
\end{array}\right.
$$

Observe that by lower-semicontinuity we have that for any $q \geq 1$ there holds

$$
\left(\iint_{\Omega_{T}}|D u|^{q} \mathrm{~d} x \mathrm{~d} t\right)^{\frac{1}{q}} \leq \liminf _{j \rightarrow \infty}\left(\iint_{\Omega_{T}}\left|D u_{j}\right|^{q} \mathrm{~d} x \mathrm{~d} t\right)^{\frac{1}{q}} \leq L,
$$

so that $\|D u\|_{L^{\infty}\left(\Omega_{T}, \mathbb{R}^{n}\right)} \leq L$. Turning our attention to the initial condition, we first observe from (5.3) and (5.4) that

$$
\left\|u_{j}\left(t_{2}\right)-u_{j}\left(t_{1}\right)\right\|_{L^{2}(\Omega)}^{2} \leq 4 \mathrm{e}^{\frac{T}{\varepsilon}}|\Omega| \sup _{B_{L}(0)}|f|\left(t_{2}-t_{1}\right)
$$

holds true for any $0 \leq t_{1}<t_{2} \leq T$. Recalling that $u_{j}(0)=u_{o}$, the last estimate with $t_{1}=0$ and the weak convergence $u_{j} \rightarrow u$ in $L^{2}\left(\Omega_{T}\right)$ imply

$$
\frac{1}{h} \int_{0}^{h}\left\|u(t)-u_{o}\right\|_{L^{2}(\Omega)}^{2} \mathrm{~d} t \leq \liminf _{j \rightarrow \infty} \frac{1}{h} \int_{0}^{h}\left\|u_{j}(t)-u_{o}\right\|_{L^{2}(\Omega)}^{2} \mathrm{~d} t \leq 4 \mathrm{e}^{\frac{T}{\varepsilon}}|\Omega| \sup _{B_{L}(0)}|f| h .
$$

This, however, yields

$$
\lim _{h \downarrow 0} \frac{1}{h} \int_{0}^{h}\left\|u(t)-u_{o}\right\|_{L^{2}(\Omega)}^{2} \mathrm{~d} t=0,
$$


so that $u(0)=u_{o}$ in the usual $L^{2}$-sense. Finally, the limit map $u$ fulfills on the lateral boundary $\partial_{\mathcal{P}} \Omega_{T}$ the condition that $u(t)=u_{o}$ for almost every $t \in(0, T)$ in the sense of traces in $W^{1, q}(\Omega)$, for any $q \geq 1$. On the other hand, since $u(t) \in W^{1, \infty}(\Omega)$ for a.e. $t \in(0, T)$, for those good time slices $u(t)$ can be uniquely extended to $\bar{\Omega}$, which means that $u(t)$ admits a trace in the usual sense. This trace has of course to coincide with the trace of $u_{o}$, proving that $u(t)=u_{o}$ on $\partial \Omega$ for a.e. $t$. All together we conclude that $u \in \widehat{K}_{u_{o}}^{(L)}\left(\Omega_{T}\right)$, and it remains to prove that $u$ is indeed the unique minimizer of $F_{\varepsilon}$ in the class $\widehat{K}_{u_{o}}^{(L)}\left(\Omega_{T}\right)$. This, however, follows by means of lower semicontinuity arguments for the functional $F_{\varepsilon}$ with respect to the convergences above, i.e. by establishing

$$
F_{\varepsilon}(u) \leq \liminf _{j \rightarrow \infty} F_{\varepsilon}\left(u_{j}\right)=\lim _{j \rightarrow \infty} F_{\varepsilon}\left(u_{j}\right)=\inf _{v \in \widehat{K}_{u_{o}}^{(L)}\left(\Omega_{T}\right)} F_{\varepsilon}(v) .
$$

Using the weak convergence $\partial_{t} u_{j} \rightarrow \partial_{t} u$ in $L^{2}\left(\Omega_{T}\right)$ we conclude that

$$
\iint_{\Omega_{T}} \mathrm{e}^{-\frac{t}{\varepsilon}}\left|\partial_{t} u\right|^{2} \mathrm{~d} x \mathrm{~d} t \leq \liminf _{j \rightarrow \infty} \iint_{\Omega_{T}} \mathrm{e}^{-\frac{t}{\varepsilon}}\left|\partial_{t} u_{j}\right|^{2} \mathrm{~d} x \mathrm{~d} t .
$$

Here, we used [14, Thm. 4.3]. The argument concerning the integral involving the spatial gradient $D v$ is more involved, however. Since $f$ is convex on $\mathbb{R}^{n}$, there exists a Borel-vectorfield $\lambda: \mathbb{R}^{n} \rightarrow \mathbb{R}^{n}$, bounded on compact subsets of $\mathbb{R}^{n}$, such that

$$
f(w) \geq f(\xi)+\lambda(\xi) \cdot(w-\xi) \quad \forall w, \xi \in \mathbb{R}^{n} .
$$

Therefore, we have

$$
\iint_{\Omega_{T}} \mathrm{e}^{-\frac{t}{\varepsilon}} f\left(D u_{j}\right) \mathrm{d} x \mathrm{~d} t \geq \iint_{\Omega_{T}} \mathrm{e}^{-\frac{t}{\varepsilon}}\left[f(D u)+\lambda(D u) \cdot\left(D u_{j}-D u\right)\right] \mathrm{d} x \mathrm{~d} t .
$$

Turning to the second term on the right-hand side of the preceding inequality, we observe that $\Omega_{T} \ni(x, t) \mapsto$ $\mathrm{e}^{-\frac{t}{\varepsilon}} \lambda(D u(x, t)) \in \mathbb{R}^{n}$ is bounded and measurable. Therefore, by the weak convergence $D u_{j} \rightarrow D u$ in $L^{q}\left(\Omega_{T}, \mathbb{R}^{n}\right)$ for any $q \geq 1$ we infer

$$
\lim _{j \rightarrow \infty} \iint_{\Omega_{T}} \mathrm{e}^{-\frac{t}{\varepsilon}} \lambda(D u) \cdot\left(D u_{j}-D u\right) \mathrm{d} x \mathrm{~d} t=0 .
$$

But this implies

$$
\liminf _{j \rightarrow \infty} \iint_{\Omega_{T}} \mathrm{e}^{-\frac{t}{\varepsilon}} f\left(D u_{j}\right) \mathrm{d} x \mathrm{~d} t \geq \iint_{\Omega_{T}} \mathrm{e}^{-\frac{t}{\varepsilon}} f(D u) \mathrm{d} x \mathrm{~d} t,
$$

eventually proving the first inequality in (5.7). This proves that $u$ is a minimizer of $F_{\varepsilon}$ in the class $\widehat{K}_{u_{o}}^{(L)}\left(\Omega_{T}\right)$. The uniqueness of $u$ follows, because the term involving the time derivative ensures the strict convexity of the functional $F_{\varepsilon}$.

\subsection{The minimality condition revisited}

In this section we rewrite the minimality condition of the approximations $u_{\varepsilon}$ in a form, which is more useful in the derivation of uniform bounds for the time derivative and in the limit procedure $\varepsilon \downarrow 0$. For fixed $\varepsilon \in(0,1]$ consider testing functions $\varphi \in L^{\infty}\left(\Omega_{T}\right)$ with $D \varphi \in L^{\infty}\left(\Omega_{T}, \mathbb{R}^{n}\right)$ and $\partial_{t} \varphi \in L^{2}\left(\Omega_{T}\right)$, vanishing on the lateral boundary $\partial \Omega \times(0, T)$, and satisfying

$$
\sup _{\Omega_{T}}\left|D u_{\varepsilon}+D \varphi\right| \leq L \text {. }
$$

Moreover, let $\xi \in W^{1, \infty}(0, T)$ with $0 \leq \xi \leq 1$, and $\delta \in\left(0, \mathrm{e}^{-\frac{T}{\varepsilon}}\right]$. Further, define $\sigma(t):=\delta \mathrm{e}^{\frac{t}{\varepsilon}} \xi(t)$ as well as

$$
\tilde{\varphi}_{\varepsilon, \delta}(x, t):=\sigma(t) \varphi(x, t) \equiv \delta \mathrm{e}^{\frac{t}{\varepsilon}} \xi(t) \varphi(x, t), \quad(x, t) \in \Omega_{T},
$$

while assuming either $\xi(0)=0$ or $\varphi(0)=0$. Then, set 


$$
v_{\varepsilon, \delta}(x, t):=u_{\varepsilon}(x, t)+\tilde{\varphi}_{\varepsilon, \delta}(x, t) \equiv u_{\varepsilon}(x, t)+\delta \mathrm{e}^{\frac{t}{\varepsilon}} \xi(t) \varphi(x, t)
$$

and observe that $v_{\varepsilon, \delta} \in L^{\infty}\left(\Omega_{T}\right), D v_{\varepsilon, \delta} \in L^{\infty}\left(\Omega_{T}, \mathbb{R}^{n}\right)$ and $\partial_{t} v_{\varepsilon, \delta} \in L^{2}\left(\Omega_{T}\right)$. Further, $v_{\varepsilon, \delta}=u_{o}$ on the parabolic boundary $\partial_{\mathcal{P}} \Omega_{T}$. To conclude $v_{\varepsilon, \delta} \in \widehat{K}_{u_{o}}^{(L)}\left(\Omega_{T}\right)$, i.e. that it is an admissible comparison map in the minimality condition for $u_{\varepsilon}$, it remains to show that $\sup _{\Omega_{T}}\left|D v_{\varepsilon, \delta}\right| \leq L$. But, this follows from the fact that $v_{\varepsilon, \delta}$ is a convex combination of $u_{\varepsilon}$ and $u_{\varepsilon}+\varphi$ on every fixed time slice $t \in[0, T]$, the assumption (5.8) and the fact that $0 \leq \sigma(t) \leq 1$ by the choice of $\delta$ above. The minimality of $u_{\varepsilon}$ thus shows that

$$
F_{\varepsilon}\left(u_{\varepsilon}\right) \leq F_{\varepsilon}\left(v_{\varepsilon, \delta}\right)
$$

which by the convexity of $f$ can be rewritten to

$$
\begin{aligned}
\frac{\delta}{\varepsilon} \iint_{\Omega_{T}} \xi(t) f\left(D u_{\varepsilon}\right) \mathrm{d} x \mathrm{~d} t & \leq \iint_{\Omega_{T}} \mathrm{e}^{-\frac{t}{\varepsilon}}\left[\frac{1}{2} \delta^{2}\left|\partial_{t}\left(\mathrm{e}^{\frac{t}{\varepsilon}} \xi \varphi\right)\right|^{2}+\delta \partial_{t} u_{\varepsilon} \partial_{t}\left(\mathrm{e}^{\frac{t}{\varepsilon}} \xi \varphi\right)\right] \mathrm{d} x \mathrm{~d} t \\
& +\frac{\delta}{\varepsilon} \iint_{\Omega_{T}} \xi(t) f\left(D u_{\varepsilon}+D \varphi\right) \mathrm{d} x \mathrm{~d} t
\end{aligned}
$$

Multiplying both sides of the previous inequality by $\varepsilon / \delta$ and letting $\delta \downarrow 0$, yields

$$
\begin{aligned}
\iint_{\Omega_{T}} \xi(t) f\left(D u_{\varepsilon}\right) \mathrm{d} x \mathrm{~d} t \leq & \iint_{\Omega_{T}} \xi(t) f\left(D u_{\varepsilon}+D \varphi\right) \mathrm{d} x \mathrm{~d} t+\iint_{\Omega_{T}} \xi \partial_{t} u_{\varepsilon} \varphi \mathrm{d} x \mathrm{~d} t \\
& +\varepsilon \iint_{\Omega_{T}}\left[\xi^{\prime} \partial_{t} u_{\varepsilon} \varphi+\xi \partial_{t} u_{\varepsilon} \partial_{t} \varphi\right] \mathrm{d} x \mathrm{~d} t
\end{aligned}
$$

for any $\varphi \in L^{\infty}\left(\Omega_{T}\right)$ with $D \varphi \in L^{\infty}\left(\Omega_{T}, \mathbb{R}^{n}\right)$ and $\partial_{t} \varphi \in L^{2}\left(\Omega_{T}\right)$, vanishing on the lateral boundary $\partial \Omega \times(0, T)$, satisfying (5.8), and for any $\xi \in W^{1, \infty}((0, T))$ with $0 \leq \xi \leq 1$, and such that either $\xi(0)=0$ or $\varphi(0)=0$.

\subsection{A uniform estimate for the time derivative}

In this section we shall establish a uniform $L^{2}$-bound for the time derivative of $F_{\varepsilon}$-minimizers $u_{\varepsilon} \in \widehat{K}_{u_{o}}^{(L)}\left(\Omega_{T}\right)$. This estimate together with the bound

$$
\sup _{0<\varepsilon \leq 1}\left[\left\|u_{\varepsilon}\right\|_{L^{\infty}\left(\Omega_{T}\right)}+\left\|D u_{\varepsilon}\right\|_{L^{\infty}\left(\Omega_{T}, \mathbb{R}^{n}\right)}\right] \leq\left\|u_{o}\right\|_{L^{\infty}(\Omega)}+L(1+\operatorname{diam}(\Omega))
$$

allows the extraction of a converging subsequence in the limit $\varepsilon \downarrow 0$. Note that (5.10) follows from (5.2). To this end, define $\left[u_{\varepsilon}\right]_{h}$ according to (2.1) with $u_{o}$ and $u_{\varepsilon}$ instead of $v_{o}$ and $v$, respectively. We can use the definition with $r=q$ and $X=W^{1, q}(\Omega)$, since $u_{\varepsilon} \in L^{q}\left(0, T ; W_{u_{o}}^{1, q}(\Omega)\right)$ and $u_{o} \in W^{1, q}(\Omega)$ for any choice of $q \geq 1$. By means of Lemma 2.4 we conclude that $\left[u_{\varepsilon}\right]_{h} \in L^{q}\left(0, T ; W_{u_{o}}^{1, q}(\Omega)\right)$ with $\partial_{t}\left[u_{\varepsilon}\right]_{h} \in L^{2}\left(\Omega_{T}\right)$ and $\partial_{t}\left[u_{\varepsilon}\right]_{h}=\frac{1}{h}\left(u_{\varepsilon}-\left[u_{\varepsilon}\right]_{h}\right)$. The last identity implies even more, as the right-hand side is an element of the space $L^{q}\left(0, T ; W_{0}^{1, q}(\Omega)\right)$ whose time derivative is an element of $L^{2}\left(\Omega_{T}\right)$. Moreover, since $\left[u_{\varepsilon}\right]_{h}(0)=u_{o}$ it follows that $\partial_{t}\left[u_{\varepsilon}\right]_{h}(0)=\frac{1}{h}\left(u_{o}-\left[u_{\varepsilon}\right]_{h}(0)\right)=0$. Further, from (2.1) we conclude that

$$
\begin{aligned}
\left\|D\left[u_{\varepsilon}\right]_{h}(t)\right\|_{L^{q}(\Omega)} & \leq \mathrm{e}^{-\frac{t}{h}}\left\|D u_{o}\right\|_{L^{q}(\Omega)}+\frac{1}{h} \int_{0}^{t} \mathrm{e}^{\frac{s-t}{h}}\left\|D u_{\varepsilon}(s)\right\|_{L^{q}(\Omega)} \mathrm{d} t \\
& \leq L|\Omega|^{\frac{1}{q}}\left[\mathrm{e}^{-\frac{t}{h}}+\frac{1}{h} \int_{0}^{t} \mathrm{e}^{\frac{s-t}{h}} \mathrm{~d} s\right]=L|\Omega|^{\frac{1}{q}},
\end{aligned}
$$

establishing that

$$
\left[\iint_{\Omega_{T}}\left|D\left[u_{\varepsilon}\right]_{h}\right|^{q} \mathrm{~d} x \mathrm{~d} t\right]^{\frac{1}{q}} \leq L \quad \forall q \geq 1 .
$$


But this implies $\left\|D\left[u_{\varepsilon}\right]_{h}\right\|_{L^{\infty}\left(\Omega_{T}, \mathbb{R}^{n}\right)} \leq L$, and we conclude that

$$
\left\|D\left(u_{\varepsilon}-h \partial_{t}\left[u_{\varepsilon}\right]_{h}\right)\right\|_{L^{\infty}\left(\Omega_{T}, \mathbb{R}^{n}\right)}=\left\|D\left[u_{\varepsilon}\right]_{h}\right\|_{L^{\infty}\left(\Omega_{T}, \mathbb{R}^{n}\right)} \leq L .
$$

In other words, we are allowed to take $\varphi=-h \partial_{t}\left[u_{\varepsilon}\right]_{h}$ as testing function in (5.9) (note that $\varphi(0)=0$ ), which for any $\xi \in W^{1, \infty}(0, T)$ with $0 \leq \xi \leq 1$ implies

$$
\begin{aligned}
h \iint_{\Omega_{T}}\left[\left(\xi+\varepsilon \xi^{\prime}\right) \partial_{t} u_{\varepsilon} \partial_{t}\left[u_{\varepsilon}\right]_{h}+\varepsilon \xi \partial_{t} u_{\varepsilon} \partial_{t t}\left[u_{\varepsilon}\right] h\right] \mathrm{d} x \mathrm{~d} t \\
\leq \iint_{\Omega_{T}} \xi(t)\left[f\left(D\left[u_{\varepsilon}\right]_{h}\right)-f\left(D u_{\varepsilon}\right)\right] \mathrm{d} x \mathrm{~d} t \\
\leq \iint_{\Omega_{T}} \xi(t)\left[\left[f\left(D u_{\varepsilon}\right)\right]_{h}-f\left(D u_{\varepsilon}\right)\right] \mathrm{d} x \mathrm{~d} t \\
=-h \iint_{\Omega_{T}} \xi(t) \partial_{t}\left[f\left(D u_{\varepsilon}\right)\right]_{h} \mathrm{~d} x \mathrm{~d} t,
\end{aligned}
$$

by means of Lemma 2.5, where $\left[f\left(u_{\varepsilon}\right)\right]_{h}$ is defined according to (2.1) with $v_{o}$ and $v$ replaced by $f\left(D u_{o}\right)$ and $f\left(D u_{\varepsilon}\right)$, respectively. The second term on the left-hand side of the previous inequality can be estimated further as follows

$$
\begin{aligned}
\partial_{t} u_{\varepsilon} \partial_{t t}\left[u_{\varepsilon}\right]_{h} & =\partial_{t}\left[u_{\varepsilon}\right]_{h} \partial_{t t}\left[u_{\varepsilon}\right]_{h}+\left(\partial_{t} u_{\varepsilon}-\partial_{t}\left[u_{\varepsilon}\right]_{h}\right) \partial_{t t}\left[u_{\varepsilon}\right]_{h} \\
& =\frac{1}{2} \partial_{t}\left|\partial_{t}\left[u_{\varepsilon}\right]_{h}\right|^{2}+\frac{1}{h}\left|\partial_{t}\left[u_{\varepsilon}\right]_{h}-\partial_{t} u_{\varepsilon}\right|^{2} \\
& \geq \frac{1}{2} \partial_{t}\left|\partial_{t}\left[u_{\varepsilon}\right]_{h}\right|^{2} .
\end{aligned}
$$

Inserting this estimate in the inequality above and dividing by $h>0$, we get

$$
\begin{array}{r}
\iint_{\Omega_{T}}\left[\left(\xi+\varepsilon \xi^{\prime}\right) \partial_{t} u_{\varepsilon} \partial_{t}\left[u_{\varepsilon}\right]_{h}+\frac{\varepsilon}{2} \xi \partial_{t}\left|\partial_{t}\left[u_{\varepsilon}\right]_{h}\right|^{2}\right] \mathrm{d} x \mathrm{~d} t \\
\leq-\iint_{\Omega_{T}} \xi(t) \partial_{t}\left[f\left(D u_{\varepsilon}\right)\right]_{h} \mathrm{~d} x \mathrm{~d} t .
\end{array}
$$

We choose $\xi \equiv 1$ in (5.11) to obtain by means of Fubini's theorem

$$
\begin{aligned}
\iint_{\Omega_{T}} \partial_{t} u_{\varepsilon} \partial_{t}\left[u_{\varepsilon}\right]_{h} \mathrm{~d} x \mathrm{~d} t & \leq-\iint_{\Omega_{T}} \partial_{t}\left[f\left(D u_{\varepsilon}\right)\right]_{h} \mathrm{~d} x \mathrm{~d} t-\frac{\varepsilon}{2} \iint_{\Omega_{T}} \partial_{t}\left|\partial_{t}\left[u_{\varepsilon}\right] h\right|^{2} \mathrm{~d} x \mathrm{~d} t \\
& =\int_{\Omega}\left[f\left(D u_{\varepsilon}\right)\right]_{h}(0) \mathrm{d} x-\iint_{\Omega}\left[f\left(D u_{\varepsilon}\right)\right]_{h}(T) \mathrm{d} x \\
& \quad+\frac{\varepsilon}{2} \int_{\Omega}\left(\left|\partial_{t}\left[u_{\varepsilon}\right]_{h}\right|^{2}(0)-\left|\partial_{t}\left[u_{\varepsilon}\right]_{h}\right|^{2}(T)\right) \mathrm{d} x \\
& \leq 2|\Omega| \sup _{B_{L}(0)}|f| .
\end{aligned}
$$

In the last inequality we also used the facts $\partial_{t}\left[u_{\varepsilon}\right]_{h}(0)=0,\left|\partial_{t}\left[u_{\varepsilon}\right]_{h}\right|^{2}(T) \geq 0$ and the obvious upper bounds for $\left|\left[f\left(D u_{\varepsilon}\right)\right]_{h}(0)\right|$ and $\left|\left[f\left(D u_{\varepsilon}\right)\right]_{h}(T)\right|$. Taking into account that $\partial_{t}\left[u_{\varepsilon}\right]_{h} \rightarrow \partial_{t} u_{\varepsilon}$ in $L^{2}\left(\Omega_{T}\right)$ due to Lemma 2.4 , the last inequality therefore implies the following uniform bound on the time derivative

$$
\iint_{\Omega_{T}}\left|\partial_{t} u_{\varepsilon}\right|^{2} \mathrm{~d} x \mathrm{~d} t \leq 2|\Omega| \sup _{B_{L}(0)}|f| .
$$

Moreover, due to (5.3) and the bound this implies 


$$
\left\|u_{\varepsilon}\left(t_{2}\right)-u_{\varepsilon}\left(t_{1}\right)\right\|_{L^{2}(\Omega)} \leq\left(2|\Omega| \sup _{B_{L}(0)}|f|\right)^{\frac{1}{2}} \sqrt{\left|t_{2}-t_{1}\right|}
$$

for any $t_{1}, t_{2} \in[0, T]$. In other words, (5.13) implies that the family $\left(u_{\varepsilon}\right)_{\varepsilon \in(0,1]}$ of minimizers is also uniformly bounded in $C^{0, \frac{1}{2}}\left([0, T] ; L^{2}(\Omega)\right)$.

\section{Existence of solutions to the gradient constrained problem}

In this section we establish the existence of variational solutions to the gradient constrained problem in the sense of Definition 3.1. More precisely, we show:

Theorem 6.1 (Existence to the gradient constrained problem). Let $L>0$ and $\Omega$ be a bounded, open set in $\mathbb{R}^{n}$. Further, suppose that $f: \Omega \rightarrow \mathbb{R}$ is a convex function and that $u_{o} \in W^{1, \infty}(\Omega)$ with $\left\|D u_{o}\right\|_{L^{\infty}\left(\Omega, \mathbb{R}^{n}\right)} \leq L$. Then, for any $T \in(0, \infty]$ there exists a unique variational solution $u$ on $\Omega_{T}$ in the class $K_{u_{o}}^{(L)}\left(\Omega_{T}\right)$ in the sense of Definition 3.1.

Proof. First, we assume $T<\infty$. For any $\varepsilon \in(0,1]$ we denote by $u_{\varepsilon}$ the unique $F_{\varepsilon}$-minimizer from Lemma 5.1 on $\Omega_{T}$. In the following we will perform the limit procedure $\varepsilon \downarrow 0$ in the sequence $\left(u_{\varepsilon}\right)_{\varepsilon>0}$. By means of the estimates (5.10), (5.12), and (5.13) the sequence $\left(u_{\varepsilon}\right)_{\varepsilon>0}$ is uniformly bounded in the spaces $L^{\infty}\left(\Omega_{T}\right)$ and $C^{0, \frac{1}{2}}\left([0, T] ; L^{2}(\Omega)\right)$, the sequence of spatial gradients $\left(D u_{\varepsilon}\right)_{\varepsilon>0}$ is uniformly bounded in $L^{\infty}\left(\Omega_{T}, \mathbb{R}^{n}\right)$, and the sequence of the corresponding time derivatives $\left(\partial_{t} u_{\varepsilon}\right)_{\varepsilon>0}$ is uniformly bounded in the space $L^{2}\left(\Omega_{T}\right)$. Therefore, there exists a map

$$
u \in \bigcap_{q \geq 1} L^{q}\left(0, T ; W^{1, p}(\Omega)\right) \quad \text { with } \quad \partial_{t} u \in L^{2}\left(\Omega_{T}\right)
$$

and a subsequence of $u_{\varepsilon}$ (still denoted this way) such that

$$
\left\{\begin{array}{c}
u_{\varepsilon} \rightarrow u \quad \text { weakly in } L^{q}\left(\Omega_{T}\right) \text { for any } q \geq 1, \\
D u_{\varepsilon} \rightarrow D u \text { weakly in } L^{q}\left(\Omega_{T}, \mathbb{R}^{n}\right) \text { for any } q \geq 1, \\
\partial_{t} u_{\varepsilon} \rightarrow \partial_{t} u \text { weakly in } L^{2}\left(\Omega_{T}\right) .
\end{array}\right.
$$

Moreover, from above convergences and the compactness result in [31] we infer the existence of a further (not relabeled) subsequence such that

$$
u_{\varepsilon} \rightarrow u \quad \text { strongly in } L^{2}\left(\Omega_{T}\right) .
$$

Note that the above convergences together with the fact that $u_{\varepsilon}(0)=u_{o}$ for any $\varepsilon \in(0,1]$ imply by a similar argument as in the proof of Lemma 5.1 that $u \in C^{0, \frac{1}{2}}\left([0, T] ; L^{2}(\Omega)\right)$ with $u(0)=u_{o}$. Moreover, the argument from Lemma 5.1 leading to the validity of the boundary condition on the lateral boundary $\partial \Omega \times(0, T)$ also applies in the context here, eventually proving the boundary condition $u=u_{0}$ on $\partial_{\mathcal{P}} \Omega_{T}$. By lower semicontinuity with respect to weak $L^{2}$-convergence and the uniform bound (5.12) it holds that

$$
\iint_{\Omega_{T}}\left|\partial_{t} u\right|^{2} \mathrm{~d} x \mathrm{~d} t \leq \liminf _{\varepsilon \downarrow 0} \iint_{\Omega_{T}}\left|\partial_{t} u_{\varepsilon}\right|^{2} \mathrm{~d} x \mathrm{~d} t \leq 2|\Omega| \sup _{B_{L}(0)}|f| .
$$

Moreover, the lower semicontinuity of the $L^{q}$-norm with respect to weak convergence and the fact that $\left\|D u_{\varepsilon}\right\|_{L^{\infty}\left(\Omega_{T}, \mathbb{R}^{n}\right)} \leq L$, lead as in the proof of (5.6) to the assertion that also $\|D u\|_{L^{\infty}\left(\Omega_{T}, \mathbb{R}^{n}\right)} \leq L$ holds true.

Next, turning to the lower semicontinuity of the integral related to the integrand $f$, we observe that, thanks to the convexity of $f$, there exists a Borel-vectorfield $\lambda: \mathbb{R}^{n} \rightarrow \mathbb{R}^{n}$, bounded on compact subsets of $\mathbb{R}^{n}$, such that

$$
f(w) \geq f(\xi)+\lambda(\xi) \cdot(w-\xi) \quad \forall w, \xi \in \mathbb{R}^{n} .
$$

Therefore, we have

$$
\iint_{\Omega_{T}} f\left(D u_{\varepsilon}\right) \mathrm{d} x \mathrm{~d} t \geq \iint_{\Omega_{T}}\left[f(D u)+\lambda(D u) \cdot\left(D u_{\varepsilon}-D u\right)\right] \mathrm{d} x \mathrm{~d} t .
$$


The second term on the right-hand side of the preceding inequality vanishes in the limit $\varepsilon \downarrow 0$, since $\Omega_{T} \ni(x, t) \mapsto$ $\lambda(D u(x, t)) \in \mathbb{R}^{n}$ is bounded and measurable and $D u_{\varepsilon} \rightarrow D u$ in $L^{q}\left(\Omega_{T}\right)$ for any $q \geq 1$. That is,

$$
\lim _{j \rightarrow \infty} \iint_{\Omega_{T}} \lambda(D u) \cdot\left(D u_{\varepsilon}-D u\right) \mathrm{d} x \mathrm{~d} t=0 .
$$

Inserting this above, we arrive at

$$
\liminf _{j \rightarrow \infty} \iint_{\Omega_{T}} f\left(D u_{\varepsilon}\right) \mathrm{d} x \mathrm{~d} t \geq \iint_{\Omega_{T}} f(D u) \mathrm{d} x \mathrm{~d} t .
$$

Note that

$$
\left|\iint_{\Omega_{T}} f(D u) \mathrm{d} x \mathrm{~d} t\right| \leq T|\Omega| \sup _{B_{L}(0)}|f| .
$$

For $u$ to be a variational solution as in Definition 3.1 it just remains to show, that $u$ satisfies the minimality condition (3.1). To this end, consider $v \in L^{\infty}\left(\Omega_{T}\right)$ with $D v \in L^{\infty}\left(\Omega_{T}, \mathbb{R}^{n}\right)$ and $\partial_{t} v \in L^{2}\left(\Omega_{T}\right)$, such that $v=u_{o}$ on the lateral boundary and

$$
\|D v\|_{L^{\infty}\left(\Omega_{T}\right)} \leq L .
$$

For fixed $\theta \in(0, T / 2)$ let

$$
\xi_{\theta}(t):=\frac{t}{\theta} \chi_{[0, \theta]}(t)+\chi_{(\theta, T-\theta)}(t)+\frac{T-t}{\theta} \chi_{[T-\theta, T]}(t)
$$

denote a cut-off function with respect to time. Now, fix $\varepsilon \in(0,1]$ and consider $\varphi=v-u_{\varepsilon}$. The properties of $v$ and $u_{\varepsilon}$ imply that $\varphi \in L^{\infty}\left(\Omega_{T}\right)$ with $D \varphi \in L^{\infty}\left(\Omega_{T}, \mathbb{R}^{n}\right)$ and $\partial_{t} \varphi \in L^{2}\left(\Omega_{T}, \mathbb{R}^{N}\right)$. Observe that $u_{\varepsilon}+\varphi$ fulfills the assumption (5.8). Further, observe that $\varphi$ vanishes on the lateral boundary and that $\xi_{\theta}(0)=0$. Therefore, both $\varphi$ and $\xi=\xi_{\theta}$ are admissible in the inequality (5.9). Adding $\iint_{\Omega_{T}} f\left(D u_{\varepsilon}\right) \mathrm{d} x \mathrm{~d} t$ on both sides shows

$$
\begin{aligned}
& \iint_{\Omega_{T}} f\left(D u_{\varepsilon}\right) \mathrm{d} x \mathrm{~d} t \\
& \quad \leq \iint_{\Omega_{T}}\left(1-\xi_{\theta}\right) f\left(D u_{\varepsilon}\right) \mathrm{d} x \mathrm{~d} t+\iint_{\Omega_{T}} \xi_{\theta} \partial_{t} u_{\varepsilon}\left(v-u_{\varepsilon}\right) \mathrm{d} x \mathrm{~d} t \\
& \quad+\iint_{\Omega_{T}} \xi_{\theta} f(D v) \mathrm{d} x \mathrm{~d} t+\varepsilon \iint_{\Omega_{T}}\left[\xi_{\theta}^{\prime} \partial_{t} u_{\varepsilon}\left(v-u_{\varepsilon}\right)+\xi_{\theta} \partial_{t} u_{\varepsilon} \partial_{t}\left(v-u_{\varepsilon}\right)\right] \mathrm{d} x \mathrm{~d} t .
\end{aligned}
$$

In the following, we first pass to the limit $\varepsilon \downarrow 0$ and then let $\theta \downarrow 0$. Due to the strong convergence $u_{\varepsilon} \rightarrow u$ in $L^{2}\left(\Omega_{T}\right)$ and the weak convergence $\partial_{t} u_{\varepsilon} \rightarrow \partial_{t} u$ in $L^{2}\left(\Omega_{T}\right)$, the second integral on the right-hand side converges. Moreover, due to the uniform $L^{2}$-bounds of $\partial_{t} u_{\varepsilon}$ and $u_{\varepsilon}$ from (5.12) and (5.10), we know that the last integral on the right-hand side vanishes in the limit $\varepsilon \downarrow 0$. Finally, for the first integral on the right-hand side we use the facts that $1-\xi_{\theta}(t)=$ 0 for $t \in(\theta, T-\theta)$ and $\left\|D u_{\varepsilon}\right\|_{\infty} \leq L$. Therefore, using these facts and the lower semicontinuity of the integral $\iint_{\Omega_{T}} f(D v) \mathrm{d} x \mathrm{~d} t$ with respect to the weak convergences form (6.1) yields

$$
\iint_{\Omega_{T}} f(D u) \mathrm{d} x \mathrm{~d} t \leq \iint_{\Omega_{T}} \xi_{\theta} \partial_{t} u(v-u) \mathrm{d} x \mathrm{~d} t+\iint_{\Omega_{T}} \xi_{\theta} f(D v) \mathrm{d} x \mathrm{~d} t+2 \theta|\Omega| \sup _{B_{L}(0)}|f| .
$$

For the first term on the right-hand side we compute

$$
\begin{aligned}
\iint_{\Omega_{T}} \xi_{\theta} \partial_{t} u(v-u) \mathrm{d} x \mathrm{~d} t= & \iint_{\Omega_{T}} \xi_{\theta} \partial_{t} v(v-u) \mathrm{d} x \mathrm{~d} t \\
& +\frac{1}{2 \theta} \iint_{\Omega \times(0, \theta)}|v-u|^{2} \mathrm{~d} x \mathrm{~d} t-\frac{1}{2 \theta} \iint_{\Omega \times(T-\theta, T)}|v-u|^{2} \mathrm{~d} x \mathrm{~d} t,
\end{aligned}
$$


so that

$$
\begin{aligned}
\iint_{\Omega_{T}} f(D u) \mathrm{d} x \mathrm{~d} t \leq & \iint_{\Omega_{T}} \xi_{\theta}\left[\partial_{t} v(v-u)+f(D v)\right] \mathrm{d} x \mathrm{~d} t+2 \theta|\Omega| \sup _{B_{L}(0)}|f| \\
& +\frac{1}{2 \theta} \iint_{\Omega \times(0, \theta)}|v-u|^{2} \mathrm{~d} x \mathrm{~d} t-\frac{1}{2 \theta} \iint_{\Omega \times(T-\theta, T)}|v-u|^{2} \mathrm{~d} x \mathrm{~d} t,
\end{aligned}
$$

where $\theta \in(0, T / 2)$ is arbitrary. We may therefore pass to the limit $\theta \downarrow 0$, with the result that

$$
\begin{aligned}
\iint_{\Omega_{T}} f(D u) \mathrm{d} x \mathrm{~d} t \leq & \iint_{\Omega_{T}}\left[\partial_{t} v(v-u)+f(D v)\right] \mathrm{d} x \mathrm{~d} t \\
& +\frac{1}{2}\left\|v(0)-u_{o}\right\|_{L^{2}(\Omega)}^{2}-\frac{1}{2}\|(v-u)(T)\|_{L^{2}(\Omega)}^{2}
\end{aligned}
$$

holds true for any $v \in L^{\infty}\left(\Omega_{T}\right)$ with $D v \in L^{\infty}\left(\Omega_{T}, \mathbb{R}^{n}\right)$ and $\partial_{t} v \in L^{2}\left(\Omega_{T}\right)$, coinciding with $u_{o}$ on the lateral boundary and such that $\|D v\|_{L^{\infty}\left(\Omega_{T}\right)} \leq L$, i.e. $u$ is indeed a variational solution on $\Omega_{T}$ as in Definition 3.1. This finishes the proof of Theorem 6.1 in the case $T<\infty$. In order to construct a global variational solution on $\Omega_{\infty}$ we consider $0<T_{1}<T_{2}<\infty$ and denote by $u_{1}$ and $u_{2}$ the unique variational solutions on $\Omega_{T_{1}}$ and $\Omega_{T_{2}}$, respectively. By means of the localizing principle from Lemma 3.2 it follows that $u_{2}$ is also a variational solution on $\Omega_{T_{1}}$ which by Lemma 4.3 coincides with $u_{1}$ on $\Omega_{T_{1}}$. Therefore, taking a sequence $0<T_{1}<T_{2}<\ldots$ with $\lim _{i \rightarrow \infty} T_{i}=\infty$ this allows for a construction of a unique global variational solution.

\section{Existence of solutions to the unconstrained problem (Proof of Theorem 1.2)}

In this section we will complete the proof of Theorem 1.2. At this point, the main difficulty is to remove the gradient constraint from the variational solutions to the gradient constrained obstacle problem constructed in Theorem 6.1.

\subsection{Affine functions}

The following lemma ensures that time independent affine functions are variational solutions with respect to their own lateral boundary values.

Lemma 7.1. Let $\Omega \subset \mathbb{R}^{n}$ be open and bounded and $f: \mathbb{R}^{n} \rightarrow \mathbb{R}$ convex. Then, any affine function $w(x, t):=a+\xi \cdot x$ independent of time with $a \in \mathbb{R}$ and $\xi \in \mathbb{R}^{n}$ is a variational solution in the sense of Definition 1.1 with boundary data $u_{o}=w$ on the parabolic boundary.

Proof. Let $w(x, t):=a+\xi \cdot x$ be an affine function independent of time with $a \in \mathbb{R}$ and $\xi \in \mathbb{R}^{n}$. Observe, that $D w \equiv \xi$ and $\|D w\|_{L^{\infty}\left(\Omega_{T}, \mathbb{R}^{n}\right)}=|\xi|$. Consider $v \in K_{w}\left(\Omega_{T}\right)$ with $\partial_{t} v \in L^{2}\left(\Omega_{T}\right)$. Then, for $\varphi:=v-w$ we have that $\varphi \in K_{0}\left(\Omega_{T}\right)$. Since $f$ is convex, we find $\lambda \in \mathbb{R}^{n}$ such that $f(\xi+\eta) \geq f(\xi)+\lambda \cdot \eta$ for any $\eta \in \mathbb{R}^{n}$. We apply this with $\eta=D \varphi(x, t)$ and obtain that

$$
f(\xi+D \varphi(x, t)) \geq f(\xi)+\lambda \cdot D \varphi(x, t) \quad \forall(x, t) \in \Omega_{T} .
$$

Integrating this over $\Omega_{T}$ we end up with

$$
\iint_{\Omega_{T}} f(D w+D \varphi) \mathrm{d} x \mathrm{~d} t \geq\left|\Omega_{T}\right| f(D w)+\iint_{\Omega_{T}} \lambda \cdot D \varphi \mathrm{d} x \mathrm{~d} t=\iint_{\Omega_{T}} f(D w) \mathrm{d} x \mathrm{~d} t .
$$

In the last identity we used an integration by parts via the Gauss-Green theorem. Assuming that $\partial_{t} \varphi \in L^{2}\left(\Omega_{T}\right)$ we can utilize the identity

$$
\iint_{\Omega_{T}} \partial_{t}(w+\varphi) \varphi \mathrm{d} x \mathrm{~d} t=\frac{1}{2}\|\varphi(T)\|_{L^{2}(\Omega)}^{2}-\frac{1}{2}\|\varphi(0)\|_{L^{2}(\Omega)}^{2} .
$$

Therefore, we end up with 


$$
\begin{aligned}
\iint_{\Omega_{T}} f(D w) \mathrm{d} x \mathrm{~d} t \leq & \iint_{\Omega_{T}}\left[\partial_{t}(w+\varphi) \varphi+f(D w+D \varphi)\right] \mathrm{d} x \mathrm{~d} t \\
& +\frac{1}{2}\|\varphi(0)\|_{L^{2}(\Omega)}^{2}-\frac{1}{2}\|\varphi(T)\|_{L^{2}(\Omega)}^{2} .
\end{aligned}
$$

Since $\varphi=v-w$ we conclude that (3.1) holds for any $v \in K_{w}\left(\Omega_{T}\right)$ with $\partial_{t} v \in L^{2}\left(\Omega_{T}\right)$ coinciding with $w$ on the lateral boundary. This proves that affine functions are variational solutions of the unconstrained problem.

\subsection{A quantitative Lipschitz bound}

We consider a variational solution $u$ with initial and boundary datum $u_{o} \in W^{1, \infty}(\Omega)$ such that $\left\|D u_{o}\right\|_{L^{\infty}\left(\Omega, \mathbb{R}^{n}\right)} \leq$ $Q$ and, moreover, such that the restriction of $u_{o}$ to the boundary $\partial \Omega$ satisfies the bounded slope condition for the same parameter $Q$. We fix $x_{o} \in \partial \Omega$. Taking the affine functions $w_{x_{o}}^{ \pm}$from the bounded slope condition, we have that $w_{x_{o}}^{-} \leq u_{o} \leq w_{x_{o}}^{+}$, so that the maximum principle from Lemma 4.4 implies the estimate

$$
w_{x_{o}}^{-}(x) \leq u(x, t) \leq w_{x_{o}}^{+}(x) \quad \forall(x, t) \in \Omega_{T},
$$

so that

$$
\left|u(x, t)-u_{o}\left(x_{o}\right)\right| \leq Q\left|x-x_{o}\right| \quad \forall(x, t) \in \Omega_{T} .
$$

Since $x_{o} \in \partial \Omega$ was arbitrary, this implies that

$$
\frac{\left|u(x, t)-u_{o}\left(x_{o}\right)\right|}{\left|x-x_{o}\right|} \leq Q \quad \forall x_{o} \in \partial \Omega,(x, t) \in \Omega_{T} .
$$

Next, we consider points $x_{1}, x_{2} \in \Omega$ with $x_{1} \neq x_{2}$ and $t \in(0, T)$. We let $y:=x_{2}-x_{1}$ and define

$$
u_{y}(x, t):=u(x+y, t) \quad \text { for }(x, t) \in \widetilde{\Omega}_{T}:=\left\{(x-y, t) \in \mathbb{R}^{n+1}:(x, t) \in \Omega_{T}\right\} .
$$

Then, $u_{y}$ is a parabolic minimizer in $K^{(L)}\left(\widetilde{\Omega}_{T}\right)$ on $\widetilde{\Omega}_{T}$ in the sense of Definition 4.1. From Remark 4.2 we know that both, $u$ and $u_{y}$ are parabolic minimizers in $K^{(L)}\left((\Omega \cap \widetilde{\Omega})_{T}\right)$ on $(\Omega \cap \widetilde{\Omega})_{T}:=(\Omega \cap \widetilde{\Omega}) \times(0, T)$. Applying the maximum principle from Lemma 4.4 we conclude that there exists $\left(x_{o}, t_{o}\right) \in \partial_{\mathcal{P}}\left((\Omega \cap \tilde{\Omega})_{T}\right)$ such that

$$
\left|u\left(x_{1}, t\right)-u_{y}\left(x_{1}, t\right)\right| \leq\left|u\left(x_{o}, t_{o}\right)-u_{y}\left(x_{o}, t_{o}\right)\right| .
$$

This, together with the definition of $u_{y}$ yields

$$
\begin{aligned}
\left|u\left(x_{1}, t\right)-u\left(x_{2}, t\right)\right| & =\left|u\left(x_{1}, t\right)-u\left(x_{1}+y, t\right)\right|=\left|u\left(x_{1}, t\right)-u_{y}\left(x_{1}, t\right)\right| \\
& \leq\left|u\left(x_{o}, t_{o}\right)-u_{y}\left(x_{o}, t_{o}\right)\right|=\left|u\left(x_{o}, t_{o}\right)-u\left(x_{o}+y, t_{o}\right)\right| .
\end{aligned}
$$

Since either one of the points $x_{o}$ and $x_{o}+y$ belongs to $\partial \Omega$, or $t_{o}=0$, we can now use (7.1), respectively the fact that $\left\|D u_{o}\right\|_{L^{\infty}} \leq Q$ to further estimate

$$
\frac{\left|u\left(x_{1}, t\right)-u\left(x_{2}, t\right)\right|}{\left|x_{1}-x_{2}\right|} \leq \frac{\left|u\left(x_{o}, t_{o}\right)-u\left(x_{o}+y, t_{o}\right)\right|}{|y|} \leq Q .
$$

Since $x_{1}, x_{2} \in \Omega$ with $x_{1} \neq x_{2}$ are arbitrary, this implies that

$$
\|D u\|_{L^{\infty}\left(\Omega_{T}, \mathbb{R}^{n}\right)} \leq Q .
$$

\subsection{Removing the gradient constraint}

In this section we indicate how the gradient constraint hypothesis $\|D u\|_{L^{\infty}\left(\Omega, \mathbb{R}^{n}\right)} \leq L<\infty$ for a variational solution $u$ of the gradient constrained obstacle problem in $K_{u_{o}}^{(L)}\left(\Omega_{T}\right)$ can be removed. Therefore, we let $Q>0$ as in $\S 7.2$ and choose $L>Q$. Then, we have that the strict inequality

$$
\|D u\|_{L^{\infty}\left(\Omega_{T}, \mathbb{R}^{n}\right)} \leq Q<L
$$

holds true. In the following we will establish that the variational inequality (3.1) satisfied by $u$ actually holds for any comparison map $w \in K_{u_{o}}^{(\infty)}\left(\Omega_{T}\right)$ with $\partial_{t} w \in L^{2}\left(\Omega_{T}\right)$. We start with the observation that $\partial_{t} u \in L^{2}\left(\Omega_{T}\right)$ by Lemma 3.4 
and $u(0)=u_{o}$ in the $L^{2}(\Omega)$-sense. Therefore, as observed in $\S 4, u$ is a parabolic minimizer in $K_{u_{o}}^{(L)}\left(\Omega_{T}\right)$ in the sense of Definition 4.1. Consider $w \in K_{u_{o}}^{(\infty)}\left(\Omega_{T}\right)$, i.e. $w \in L^{\infty}\left(\Omega_{T}\right)$ with $D w \in L^{\infty}\left(\Omega_{T}, \mathbb{R}^{n}\right)$, coinciding with $u_{o}$ on $\partial \Omega \times(0, T)$ and such that $\partial_{t} w \in L^{2}\left(\Omega_{T}\right)$. As testing function in (4.1) we choose

$$
v:=u+s(w-u) \quad \text { for } 0<s \ll 1 .
$$

Observe that this choice is allowed, since $\partial_{t} v \in L^{2}\left(\Omega_{T}\right)$ and $\|D v\|_{L^{\infty}\left(\Omega_{T}\right)}<L$ for $s>0$ small enough. Moreover, $v=u_{o}$ on the lateral boundary. From (3.1) and the convexity of $f$ we infer

$$
\begin{aligned}
\iint_{\Omega_{T}} f(D u) \mathrm{d} x \mathrm{~d} t & \leq \iint_{\Omega_{T}}\left[s \partial_{t} u(w-u)+f((1-s) D u+s D w)\right] \mathrm{d} x \mathrm{~d} t \\
& \leq \iint_{\Omega_{T}}\left[s \partial_{t} u(w-u)+(1-s) f(D u)+s f(D w)\right] \mathrm{d} x \mathrm{~d} t .
\end{aligned}
$$

We re-absorb the second term of the right-hand side in the left-hand side and divide the result by $s>0$. This leads to the inequality

$$
\begin{aligned}
\iint_{\Omega_{T}} f(D u) \mathrm{d} x \mathrm{~d} t \leq & \iint_{\Omega_{T}}\left[\partial_{t} u(w-u)+f(D w)\right] \mathrm{d} x \mathrm{~d} t \\
= & \iint_{\Omega_{T}}\left[\partial_{t} w(w-u)-\frac{1}{2} \partial_{t}|w-u|^{2}+f(D w)\right] \mathrm{d} x \mathrm{~d} t \\
= & \iint_{\Omega_{T}}\left[\partial_{t} w(w-u)+f(D w)\right] \mathrm{d} x \mathrm{~d} t \\
& +\frac{1}{2}\left\|w(0)-u_{o}\right\|_{L^{2}(\Omega)}^{2}-\frac{1}{2}\|(w-u)(T)\|_{L^{2}(\Omega)}^{2},
\end{aligned}
$$

i.e. the variational inequality (3.1) holds true for any comparison function $w \in K_{u_{o}}^{(\infty)}\left(\Omega_{T}\right)$ satisfying $\partial_{t} w \in L^{2}\left(\Omega_{T}\right)$ and therefore $u$ is a variational solution of the unconstrained problem in the sense of Definition 1.1. This finishes the proof of Theorem 1.2.

\section{Regularity (Proof of Theorem 1.3)}

The first assertion in Theorem 1.3 already follows from Lemma 3.4. Therefore, it remains to show that the variational solution $u$ is Lipschitz continuous with respect to the parabolic metric, i.e. $u \in C^{0 ; 1,1 / 2}\left(\bar{\Omega}_{T}\right)$, if $f$ is of class $C^{1}$.

A first consequence, which holds true in case of differentiable integrands, is the validity of the associated Euler-Lagrange equation. More precisely, variational solutions $u$ (recall the gradient bound $\|D u\|_{L^{\infty}\left(\Omega_{T}\right)} \leq$ $\left.\max \left\{Q,\left\|D u_{o}\right\|_{L^{\infty}(\Omega)}\right\}=: M\right)$ are weak solutions of the associated parabolic equation, i.e. we have

$$
\iint_{\Omega_{T}}\left[u \varphi_{t}-D f(D u) \cdot D \varphi\right] \mathrm{d} x \mathrm{~d} t=0 \quad \forall \varphi \in C_{0}^{\infty}\left(\Omega_{T}\right) .
$$

Using the Poincaré inequality for solutions to parabolic equations (cf. [7, Lemma 3.1]) we obtain

$$
\iint_{Q_{\varrho}\left(z_{o}\right)}\left|u-(u)_{z_{o} ; \varrho}\right|^{2} \mathrm{~d} x \mathrm{~d} t \leq C(n) \varrho^{2}\left[\int_{Q_{\varrho}\left(z_{o}\right)}|D u|^{2} \mathrm{~d} x \mathrm{~d} t+\sup _{B_{M}(0)}|D f|^{2}\right] \leq C \varrho^{2},
$$

for any parabolic cylinder $Q_{\varrho}\left(z_{o}\right):=B_{\varrho}\left(x_{o}\right) \times\left(t_{o}-\varrho^{2}, t_{o}+\rho^{2}\right) \subset \Omega_{T}$ with $z_{o}=\left(x_{o}, t_{o}\right) \in \Omega_{T}$. We note that $\Omega$ is convex by assumption, and therefore $\Omega$ is a Lipschitz domain. This allows to employ the methods from [6] to deduce similar versions of the above Poincaré inequality for parabolic cylinders with center $\left(x_{o}, t_{o}\right) \in \partial_{\mathcal{P}} \Omega_{T}$. At this stage, the parabolic version of Campanato's characterization of Hölder continuity (with respect to the parabolic metric) by Da Prato [12] implies the Lipschitz continuity of $u$ with respect to the parabolic metric, i.e. $u \in C^{0 ; 1,1 / 2}\left(\bar{\Omega}_{T}\right)$. 
Higher regularity can for instance be established, if the integrand $f: \mathbb{R}^{n} \rightarrow \mathbb{R}$ is of class $C^{2}\left(\mathbb{R}^{n}\right)$ and strictly convex in the sense that for every $R \in(0, \infty)$ there exists $0<\lambda_{R} \leq \Lambda_{R}$ such that

$$
\lambda_{R}|w|^{2} \leq\left\langle D^{2} f(\xi) w, w\right\rangle \leq \Lambda_{R}|w|^{2} \quad \forall w \in \mathbb{R}^{n}, \xi \in B_{R}(0)
$$

holds true. Under this assumption the standard difference quotient method implies $D^{2} u \in L_{\text {loc }}^{2}\left(\Omega_{T}, \mathbb{R}^{n \times n}\right)$ with the following quantitative Caccioppoli-type estimate

$$
\sup _{t_{o}-\frac{1}{4} \varrho^{2}<t<t_{t}} \int_{B_{\ell / 2}\left(x_{o}\right)}|D u|^{2} \mathrm{~d} x+\iint_{Q_{\ell / 2}\left(z_{o}\right)}\left|D^{2} u\right|^{2} \mathrm{~d} x \mathrm{~d} t \leq C \varrho^{-2} \iint_{Q_{\varrho}\left(z_{o}\right)}|D u|^{2} \mathrm{~d} x \mathrm{~d} t,
$$

for a constant $C=C\left(n, \Lambda_{M} / \lambda_{M}\right) \geq 1$. Eventually, this gives by differentiating the equation for any $\alpha \in\{1, \ldots, n\}$ that there holds

$$
\iint_{\Omega_{T}}\left[D_{\alpha} u \varphi_{t}-\left\langle D^{2} f(D u) D D_{\alpha} u, D \varphi\right\rangle\right] \mathrm{d} x \mathrm{~d} t=0 \quad \forall \varphi \in C_{0}^{\infty}\left(\Omega_{T}\right),
$$

i.e. the partial derivatives $w:=D_{\alpha} u$ solve a linear parabolic equation with measurable coefficients $\mathbf{a}(x, t):=$ $D^{2} f(D u(x, t))$ which are symmetric, bounded and coercive with constants $\lambda_{M}$ and $\Lambda_{M}$. The classical parabolic De Giorgi \& Nash \& Moser theory for linear parabolic equations $[28,29]$ therefore implies that $D_{\alpha} u \in C_{\mathrm{loc}}^{0 ; \beta, \beta / 2}\left(\Omega_{T}\right)$ for some Hölder exponent $\beta \in(0,1)$ depending on the same parameters as the constant $C$ from the Caccioppoli inequality. Higher regularity follows by standard bootstrap arguments (using the classical Schauder theory for parabolic equations in divergence form, cf. [21, Chapter IV]), provided the integrand $f$ is regular enough.

\section{Conflict of interest statement}

The authors declare that there is no conflict of interest.

\section{Acknowledgements}

V. Bögelein was supported by the DFG-Project BO3598/1-1 "Evolutionsgleichungen mit $p, q$-Wachstum" and P. Marcellini is a member of the Gruppo Nazionale per l'Analisi Matematica, la Probalità e le loro Applicazioni (GNAMPA) of the Istituto Nazionale di Alta Matematica (INdAM).

\section{References}

[1] G. Akagi, U. Stefanelli, Doubly nonlinear evolution equations as convex minimization, SIAM J. Math. Anal. 46 (3) (2014) $1922-1945$.

[2] L. Ambrosio, N. Gigli, G. Savaré, Gradient Flows in Metric Spaces and in the Space of Probability Measures, second edition, Lectures in Mathematics ETH Zürich, Birkhäuser Verlag, Basel, 2008.

[3] V. Bögelein, F. Duzaar, P. Marcellini, Parabolic systems with $p, q$-growth: a variational approach, Arch. Ration. Mech. Anal. 210 (1) (2013) 219-267.

[4] V. Bögelein, F. Duzaar, P. Marcellini, Existence of evolutionary variational solutions via the calculus of variations, J. Differ. Equ. 256 (12) (2014) 3912-3942.

[5] V. Bögelein, F. Duzaar, P. Marcellini, S. Signoriello, Nonlocal diffusion equations, J. Math. Anal. Appl. 432 (1) (2015) $398-428$.

[6] V. Bögelein, F. Duzaar, G. Mingione, The boundary regularity of nonlinear parabolic systems I, Ann. Inst. Henri Poincaré, Anal. Non Linéaire 27 (1) (2010) 201-255.

[7] V. Bögelein, F. Duzaar, G. Mingione, The regularity of general parabolic systems with degenerate diffusion, Mem. Am. Math. Soc. 221 (2013).

[8] P. Bousquet, On the lower bounded slope condition, J. Convex Anal. 14 (1) (2007) 119-136.

[9] P. Bousquet, Boundary continuity of solutions to a basic problem in the calculus of variations, Adv. Calc. Var. 3 (1) (2010) 1-27.

[10] A. Cellina, On the bounded slope condition and the validity of the Euler Lagrange equation, SIAM J. Control Optim. 40 (4) (2001-2002) 1270-1279.

[11] F. Clarke, Continuity of solutions to a basic problem in the calculus of variations, Ann. Sc. Norm. Super. Pisa, Cl. Sci. (5) 4 (3) (2005) $511-530$

[12] G. Da Prato, Spazi $\mathcal{L}^{(p, \vartheta)}(\Omega, \delta)$ e loro proprieta, Ann. Mat. Pura Appl. (4) 69 (1965) 383-392.

[13] E. De Giorgi, Conjectures concerning some evolution problems. (Italian) A celebration of John F. Nash, Jr., Duke Math. J. 81 (2) (1996) $255-268$.

[14] E. Giusti, Direct Methods in the Calculus of Variations, World Scientific, Singapore and River Edge and NJ, ISBN $9789812380432,2003$. 
[15] A. Haar, Über das Plateausche Problem, Math. Ann. 97 (1) (1927) 124-158.

[16] P. Hartman, L. Nirenberg, On spherical image maps whose Jacobians do not change sign, Am. J. Math. 81 (1959) $901-920$.

[17] P. Hartman, G. Stampacchia, On some non-linear elliptic differential-functional equations, Acta Math. 115 (1966) $271-310$.

[18] T. Ilmanen, Elliptic regularization and partial regularity for motion by mean curvature, Mem. Am. Math. Soc. 108 (520) (1994).

[19] J. Kinnunen, P. Lindqvist, Pointwise behaviour of semicontinuous supersolutions to a quasilinear parabolic equation, Ann. Mat. Pura Appl. (4) 185 (3) (2006) 411-435.

[20] A. Lichnewsky, R.M. Temam, Pseudosolutions of the time-dependent minimal surface problem, J. Differ. Equ. 30 (3) (1978) $340-364$.

[21] G.M. Lieberman, Second Order Parabolic Differential Equations, World Scientific Publishing Co., Inc., River Edge, NJ, 1996.

[22] C. Mariconda, G. Treu, Existence and Lipschitz regularity for minima, Proc. Am. Math. Soc. 130 (2) (2002) 395-404.

[23] C. Mariconda, G. Treu, Lipschitz regularity for minima without strict convexity of the Lagrangian, J. Differ. Equ. 243 (2) (2007) $388-413$.

[24] C. Mariconda, G. Treu, Hölder regularity for a classical problem of the calculus of variations, Adv. Calc. Var. 2 (4) (2009) 311-320.

[25] E. Mascolo, E. Schianchi, Existence theorems for nonconvex problems, J. Math. Pures Appl. (9) 62 (3) (1983) $349-359$.

[26] A. Mielke, U. Stefanelli, Weighted energy-dissipation functionals for gradient flows, ESAIM Control Optim. Calc. Var. 17 (1) (2011) 52-85.

[27] M. Miranda, Un teorema di esistenza e unicità per il problema dell'area minima in n variabili, Ann. Sc. Norm. Super. Pisa 19 (3) (1965) $233-249$.

[28] J. Moser, A Harnack inequality for parabolic differential equations, Commun. Pure Appl. Math. 17 (1964) 101-134.

[29] J. Moser, Correction to: "A Harnack inequality for parabolic differential equations", Commun. Pure Appl. Math. 20 (1967) $231-236$.

[30] E. Serra, P. Tilli, Nonlinear wave equations as limits of convex minimization problems: proof of a conjecture by De Giorgi, Ann. Math. (2) 175 (3) (2012) 1551-1574.

[31] J. Simon, Compact sets in the space $L^{p}(0, T ; B)$, Ann. Mat. Pura Appl. (4) 146 (1987) 65-96.

[32] G. Stampacchia, On some regular multiple integral problems in the calculus of variations, Commun. Pure Appl. Math. 16 (1963) $383-421$.

[33] W. Wieser, Parabolic Q-minima and minimal solutions to variational flow, Manuscr. Math. 59 (1) (1987) 63-107. 